ARTICLE

DOI: $10.1038 / s 41467-018-06003-9$

\title{
AXER is an ATP/ADP exchanger in the membrane of the endoplasmic reticulum
}

\author{
Marie-Christine Klein ${ }^{1}$, Katharina Zimmermann ${ }^{2}$, Stefan Schorr ${ }^{1}$, Martina Landini ${ }^{3}$, Patrick A.W. Klemens ${ }^{3}$, \\ Jacqueline Altensell ${ }^{3}$, Martin Jung (10 1, Elmar Krause4, Duy Nguyen ${ }^{5}$, Volkhard Helms (1) 5, Jens Rettig ${ }^{4}$, \\ Claudia Fecher-Trost ${ }^{6}$, Adolfo Cavalié ${ }^{6}$, Markus Hoth (10 ${ }^{2}$, Ivan Bogeski ${ }^{2,7}$, H. Ekkehard Neuhaus ${ }^{3}$, \\ Richard Zimmermann ${ }^{1}$, Sven Lang ${ }^{1} \&$ Ilka Haferkamp ${ }^{3}$
}

To fulfill its role in protein biogenesis, the endoplasmic reticulum (ER) depends on the Hsp70-type molecular chaperone BiP, which requires a constant ATP supply. However, the carrier that catalyzes ATP uptake into the ER was unknown. Here, we report that our screen of gene expression datasets for member(s) of the family of solute carriers that are coexpressed with BiP and are ER membrane proteins identifies SLC35B1 as a potential candidate. Heterologous expression of SLC35B1 in E. coli reveals that SLC35B1 is highly specific for ATP and ADP and acts in antiport mode. Moreover, depletion of SLC35B1 from HeLa cells reduces ER ATP levels and, as a consequence, BiP activity. Thus, human SLC35B1 may provide ATP to the ER and was named AXER (ATP/ADP exchanger in the ER membrane). Furthermore, we propose an ER to cytosol low energy response regulatory axis (termed lowER) that appears as central for maintaining ER ATP supply.

\footnotetext{
${ }^{1}$ Medical Biochemistry and Molecular Biology, Saarland University, 66421 Homburg, Germany. ${ }^{2}$ Biophysics, CIPMM Saarland University, 66421 Homburg, Germany. ${ }^{3}$ Plant Physiology, Technical University Kaiserslautern, 67663 Kaiserslautern, Germany. ${ }^{4}$ Physiology, CIPMM Saarland University, 66421 Homburg, Germany. ${ }^{5}$ Center for Bioinformatics, Saarland University, 66041 Saarbrücken, Germany. ${ }^{6}$ Experimental and Clinical Pharmacology and Toxicology, Saarland University, 66421 Homburg, Germany. ${ }^{7}$ Molecular Physiology, University Medical Center, University of Göttingen, 37073 Göttingen, Germany. These authors contributed equally: Marie-Christine Klein, Katharina Zimmermann. Correspondence and requests for materials should be addressed to I.B. (email: ivan.bogeski@med.uni-goettingen.de) or to H.E.N. (email: neuhaus@rhrk.uni-kl.de) or to R.Z. (email: richard.zimmermann@uks.eu)
} 
$\mathrm{n}$ order to play its central role in protein biogenesis, the endoplasmic reticulum (ER) of nucleated cells depends on an Hsp70-type molecular chaperone, termed immunoglobulin heavy chain binding protein ( $\mathrm{BiP}$, also called glucose-regulated protein, Grp78) $)^{1,2}$. BiP is present in the ER lumen in millimolar concentration and requires a constant supply of ATP for its various functions ${ }^{3-7}$. Moreover, ATP hydrolysis by BiP generates ADP and, therefore, necessitates ADP removal from the ER. Although, ER membrane-resident ATP/ADP antiporters have been described for the plant Arabidopsis thaliana (ER-ANT1) and for the alga Phaeodactylum tricornutum (PtNTT5) ${ }^{8,9}$, these proteins do not represent the major carriers for chemical energy in the ER of these organisms. Thus, although ATP entry into the ER is strictly required for cell function, ubiquitous proteins catalyzing the corresponding ATP uptake, and the concomitant ADP release remained unknown on the molecular level.

Screening databases for solute carriers (SLCs) $)^{10,11}$ that are located in the ER membrane (GeneCards: http://www.genecards.org, The Human Protein Atlas: https://www.proteinatlas.org) and that show the same expression pattern in human tissues as BiP, which is the main ATP-consumer in the ER lumen (GenesLikeMe: https:// genecards.weizmann.ac.il/v3/index.php?path=/GenesLikeMe), focussed our attention on SLC35B1 (UniProtKB/Swiss-Prot: P78383.1). Human SLC35B1 has up to three different isoforms that are encoded by different mRNA variants. SLC35B1 is a member of the nucleotidesugar transporter family ${ }^{12-14}$, hence its synonym UDP-galactose transporter-related protein 1 (UGTrel1), and orthologs are present in diverse eukaryotes. SLC35B1 is predicted to have ten transmembrane helices and to be structurally related to members of the drug/metabolite transporter (DMT) superfamily, which also includes the amino acid transporter $S n Y d d G$, the structure of which was recently solved $^{10,11,15}$

Here, we show that heterologously expressed SLC35B1 is highly specific for ATP and ADP and operates in antiport mode, two of four characteristics it shares with the ATP transport activity which is present in rough ER membranes. Moreover, depletion of SLC35B1 from HeLa cells reduces ER ATP levels and, therefore, BiP activity, which implies that SLC35B1 mediates ATP uptake into the ER plus ADP release from the ER in vivo. Thus SLC35B1 operates as an ATP/ADP exchanger in the ER membrane. Furthermore, we initially characterize a regulatory circuit that appears to maintain the ATP supply in the ER and is termed ER low energy response or lowER.

\section{Results}

SLC35B1 is an ER membrane protein in HeLa cells. Human SLC35B1 comes in three different isoforms (Fig. 1a) and is predicted to have ten transmembrane helices (Fig. 1b). First, we confirmed the ER localization of SLC35B1 by demonstrating the presence of SLC35B1/Isoform 2 (NM_005827.2) by immunoblot with a specific anti-SLC35B1 antibody (Fig. 1c, red asterisk in lane 1, Supplementary Fig. 1a, b) in a highly enriched membrane protein extract from pancreatic rough microsomes, which are routinely used for the analysis of ER protein import ${ }^{6,7}$ and as a source of mammalian ER proteins ${ }^{16}$. Notably, the amount of extract that was needed to allow SLC35B1 detection by Western blot corresponded to $6 \mathrm{mg}$ of total microsomal protein, suggesting that SLC35B1 is not an abundant protein in canine pancreas. Therefore, it was not technically feasible to address the question of which isoform(s) is (are) present in HeLa cells, which is consistent with the low reported level of native SLC35B1 in HeLa cells ( $15 \mathrm{nM}^{17}$; Supplementary Table 1$)$.

Next, we expressed GFP-tagged SLC35B1 in HeLa cells at a moderate level (Supplementary Fig. 1c, d) and confirmed its ER localization by colocalization with the ER protein Sec62 using super-resolution microscopy (Fig. 1d). Heterologous expression in E. coli confirmed that the GFP-tag did not affect carrier activity (see below). As a caveat, we admit that it would have been desireable to have a second confirmation for ER localization of SLC35B1 in HeLa cells, e.g., by immunofluorescence microscopy after knocking in an antibody-targetable variant into the endogenous locus. However, we refrained form using this strategy under the assumption that the used strategy of transient expression allowed for finding a better compromise between expression level and detection sensitivity.

Furthermore, we expressed Myc-DDK-tagged SLC35B1/Isoform 2 in HeLa cells at a moderate level (Supplementary Fig. 1e, f) and employed immunoprecipitation in combination with subsequent mass spectrometry to address the question in which cellular compartments potential interaction partners are located. Mock-transfected cells served as negative control. SLC35B1 was efficiently immunoprecipitated from detergent solubilized HeLa cells with ANTI-FLAG M2 affinity gel and not found in the negative control immunoprecipitation (Supplementary Table 2, position 41). Among the 50 co-immunoprecipitated proteins with the highest total peptide scores we detected 26 proteins of the ER or ER-derived vesicles, 10 plasma membrane proteins, 7 mitochondrial proteins, 3 proteins of the Golgi, 2 endosomal proteins, 1 protein of the inner nuclear membrane, and 1 ribosomal protein (Supplementary Table 2). Notably, 19 of these co-immmunoprecipitated proteins were previously found to be co-immunoprecipitated with a bona fide ER protein (hSND2/ TMEM208) ${ }^{18}$, including 4 plasma membrane and 2 mitochondrial proteins. Thus, the SLC35B1 interactome also supports the conclusion that SLC35B1 is a protein of the human ER membrane. Its predominant interaction partners are major players in ER protein import (BiP, Calnexin, Oligosaccharyltransferase, Sec61 complex, TRAP complex) and/or cellular calcium homeostasis (ITPR1 and 3, SERCA2). These results are consistent with the localization of human SLC35B1 according to The Human Protein Atlas (https://www.proteinatlas.org) and with the localization of SLC35B1 in C. elegans $^{13}$, S. cerevisiae ${ }^{12}, S$. pombe $e^{12}$, and A. thaliana (AtUTr1) ${ }^{14}$.

Heterologously expressed SLC35B1 is an ATP/ADP antiporter. To test whether SLC35B1 might act as an ATP/ADP transporter, we expressed cDNAs for SLC35B1 (P78383.1, NM_005827.1) and SLC35B1/Isoform 2 (NM_005827.2) in E. coli cells, routinely used to characterize nucleotide transport proteins ${ }^{8,9,19}$. First of all, we investigated whether the recombinant proteins are integrated into the plasma membrane using Western blot analysis. Indeed, the two SLC35B1 isoforms were expressed and inserted into the bacterial membrane (Fig. 2a, b). Moreover, E. coli cells gained ATP and ADP import capacity due to the expression of these carriers (Fig. 2c-f). Non-induced control cells showed neither protein bands that reacted with the anti-SLC35B1 antibody in the membrane fractions (Fig. 2a), nor any substantial accumulation of labeled adenine nucleotides (Fig. 2c-f). The two heterologously expressed SLC35B1 isoforms are highly specific for ATP and ADP, with no competition from AMP, CTP, GTP, UTP, UDPglucose, or UDP-galactose (its putative substrate) with $\left[a^{32} \mathrm{P}\right] \mathrm{ATP}$ import (Table 1).

The fact that newly imported $\left[a^{32} \mathrm{P}\right]$ ATP could be chased from the cells by addition of an excess of unlabeled ATP already suggested that both SLC35B1 isoforms act in an antiport mode ${ }^{9}$ (Fig. 3a, b, Supplementary Fig. 2). To further substantiate this transport mechanism, membrane proteins of the respective transfected cells were solubilized in detergent and reconstituted into liposomes. Both, SLC35B1 and SLC35B1/Isoform 2 were able to facilitate the import of ATP or ADP into proteoliposomes, 
a
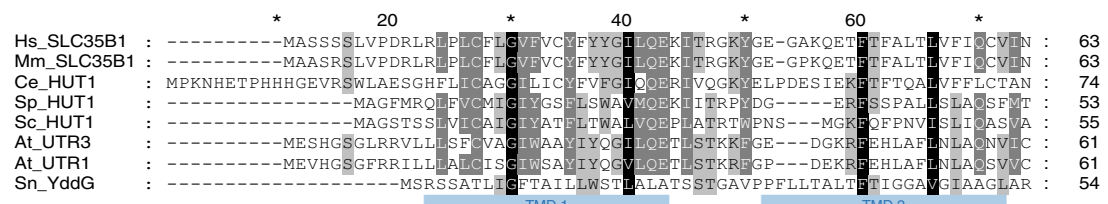

$\begin{array}{ll} & \\ \text { Hs_SLC35B1 } & : \\ \text { Mm_SLC35B1 } & : \\ \text { Ce_HUT1 } & : \\ \text { Sp_HUT1 } & : \\ \text { Sc_HUT1 } & : \\ \text { At_UTR3 } & : \\ \text { At_UTR1 } & : \\ \text { Sn_YddG } & :\end{array}$
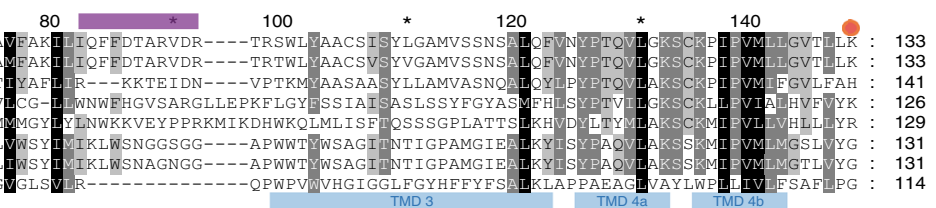

Hs_SLC35B1

Ce_HUT1

Sp_HUT1

Sc_HUT1

At_UTR1
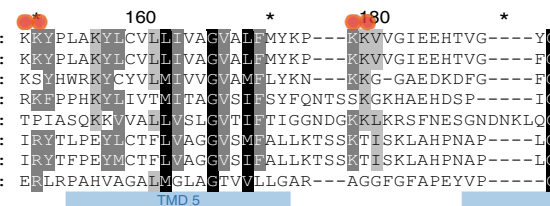

200

* 220

220

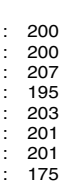

Hs_SLC35B1
Mm_SLC35B1
Ce_HUT1
Sp_HUT1
Sc_HUT1
At_UTR3
At_UTR1
Sn_YddG
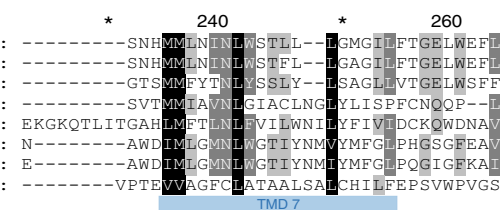

60

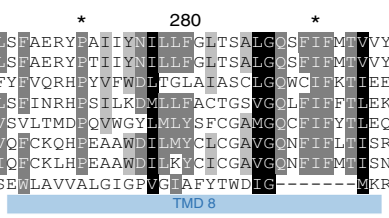

263
$: \quad 263$
$: 270$
$\vdots 258$
$: \quad 267$
$\vdots \quad 267$
$: \quad 234$

Hs_SLC35B1

Mm_SLC35B1

Me_HUT1

Sp_HUT1

Sc_HUT1

At_UTR3

Sn_YddG

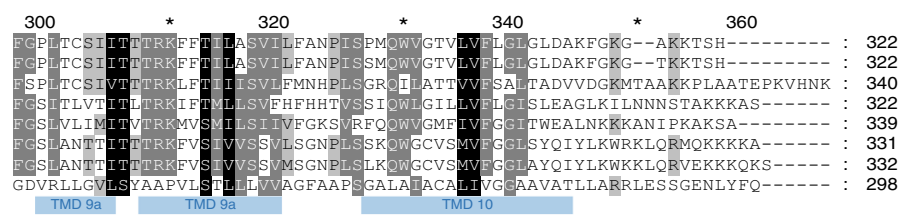

b

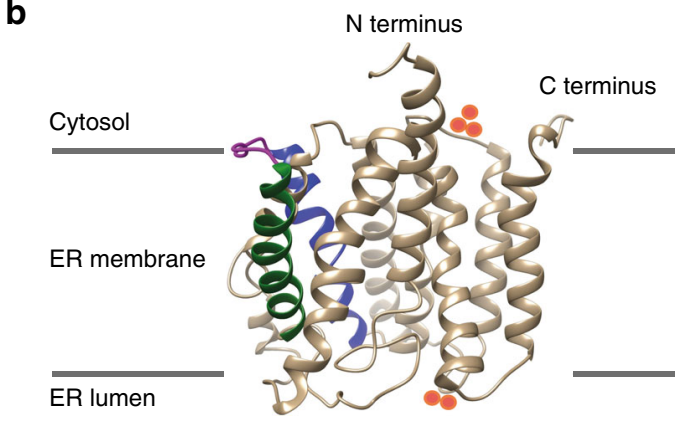

C

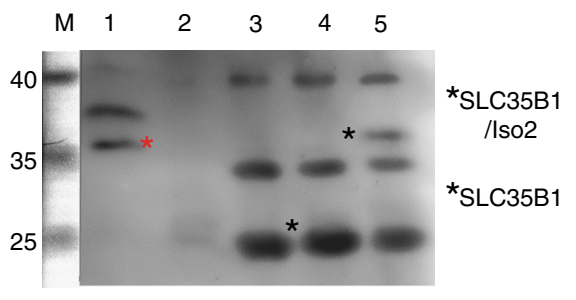

d
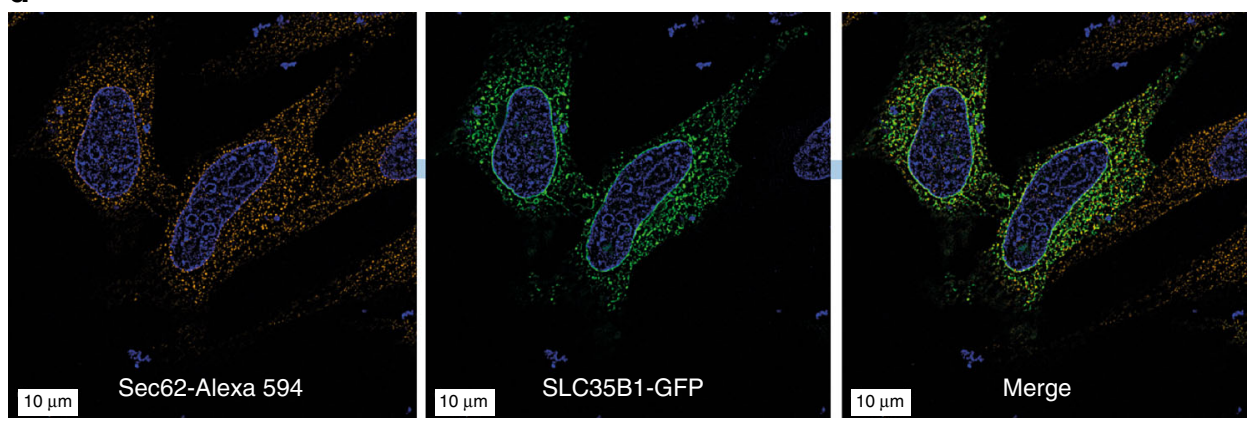

loaded with ADP or ATP (Fig. 3c-f), whereas no import was detectable in absence of loading substrates. This fact verified that the two SLC35B1 isoforms catalyze the strict antiport of ATP and ADP.

A significantly shortened variant of SLC35B1, which was reported to be encoded by transcript variant 2 (NM_001278784.1) and lacks the first two transmembrane domains (including a cluster of six aromatic amino acid residues), however, showed much lower ATP and ADP transport rates than SLC35B1 and SLC35B1/Isoform 2 (Supplementary Fig. 3a, b), although it was present in the $E$. coli membranes at similar concentrations as SLC35B1 (Fig. 2a, b). On the other hand, a heterologously expressed phosphomimetic variant of SLC35B1/Isoform 2 exhibited nearly the same biochemical characteristics as SLC35B1 and 
Fig. 1 Putative structure and intracellular localization of SLC35B1. a Protein sequences are from UniProt or GeneBank and shown in single letter code for Homo sapiens (Hs, P78383.1; NM_005827.1), Mus musculus (Mm, P97858.1), Caenorhabditis elegans (Ce, CAC35849), Schizosaccharomyces pombe (Sp, CAB46704.1), Saccharomyces cerevisiae (Sc, CAA97965), Arabidopsis thaliana (At, At1g14360 and At2g02810), and Starkeya novella (YddG, gi:502932551). The sequences were aligned using ClustalX and GeneDoc. The amino and carboxy termini face the cyosol, the double lysine motif near the carboxy terminus of mammalian SLC35B1 serves as ER retention motif. The predicted IQ motif, unique to mammalian SLC35B1, is shown in purple, positively charged clusters in red. SLC35B1/Isoform 2 comprises an amino-terminal extension of 37 amino acids (MRPLPPVGDVRLWTSPPPPLLPVPVVSGSPVGSSGRL)

(NM_005827.2), in transcript variant 2 (NM_001278784.1) the first 78 amino acids, including two N-terminal transmembrane helices, of SLC35B1 are replaced by the oligopeptide: MCDQCCVCQDL. b Hypothetical structural model of human SLC35B1, as predicted by the Phyre2 server ${ }^{34}$. Transmembrane helices 2 (green) plus 3 (blue) and the connecting loop (purple) with the putative IQ motif are highlighted, as are clusters of positively charged amino acid residues (red). c A 4\% digitonin extract of canine pancreatic rough microsomal membrane proteins (derived from 6 mg microsomal protein) was subjected to SDS-PAGE in parallel to E. coli membranes ( $25 \mu$ g protein), which were derived from non-transfected and SLC35B1-expressing or SLC35B1/isoform 2expressing cells. The Western blot was decorated with SLC35B1-specific antibody, validated in Supplementary Fig. 1a, and visualized with peroxidase-coupled secondary antibodies, Super Signal West Pico, and luminescence imaging. Molecular mass standard (M) was run in parallel and electronically copied from the stained blot to the Western blot. The relevant part of the blot is shown; the complete blot is shown in Supplementary Fig. 1b. d HeLa cells were transfected with an expression plasmid encoding SLC35B1-GFP for $8 \mathrm{~h}$, the nuclei were stained with DAPI, and the ER was visualized with Sec62-specific antibody plus Alexa-Fluor-594-coupled secondary antibody and subjected to fluorescence imaging using a super-resolution Elyra microscope ${ }^{38}$.

Representative images and merged images are shown (scale bar $10 \mu \mathrm{m}$ ). Related Western blots are shwon in Supplementary Fig. 1c, d

SLC35B1/Isoform 2 (Supplementary Fig. 3c, d), as did GFP-tagged SLC35B1 (Supplementary Fig. 3e, f), which was used for SLC35B1 visualization in HeLa cells (Fig. 1d).

SLC35B1 is similar to the ER resident ATP carrier. Heterologously expressed SLC35B1 exhibited similar apparent $K_{\mathrm{M}}$ and $V_{\max }$ values for ATP $(32.6-34.7 \mu \mathrm{M}$ and $871.0-904.5 \mathrm{pmol} \mathrm{mg}$ protein ${ }^{-1} \mathrm{~h}^{-1}$; correlation: $\left.97.5 \%\right)$ and ADP $(32.0-37.3 \mu \mathrm{M}$ and 888.4-962.3 pmol mg protein ${ }^{-1} \mathrm{~h}^{-1}$; correlation: 95.7\%) (Supplementary Fig. 4a-d), as did SLC35B1/Isoform 2 (Supplementary Fig. 4e-h). In each case, the two numbers were derived from substrate saturation experiments and corresponding EadieHofstee analyses. These apparent affinities of SLC35B1 for ATP are in line with data on ATP and ADP import into proteoliposomes, harboring the full complement of yeast ${ }^{20}$ or mammalian ER membrane proteins (Fig. 4a, Supplementary Fig. 5). In none of these eukaryotic systems, however, did the biochemical approach identify the carrier ${ }^{21-23}$. Notably, substrate specificity and antiport mode of the two SLC35B1 isoforms were also shared by the ATP/ADP carrier, which were present in proteoliposomes, harboring the full complement of mammalian ER membrane proteins (Fig. 4). ATP import into these proteoliposomes was dependent on their preloading with ADP (Fig. 4a) and not competed by AMP or other nucleoside triphosphates (Fig. 4b). Furthermore, the two heterologously expressed SLC35B1 isoforms and the ER membrane resident ATP transport activity share an insensitivity towards EDTA, i.e., transport $\mathrm{Mg}^{2+}$-free ATP (Table 1, Fig. 4b). As a caveat, we concede that it would have been ideal to additionally get the direct comparison to proteoliposmes with the full complement of ER membrane proteins from SLC35B1 over-producing HeLa cells. However, we refrained from trying this strategy assuming that it is not technically feasible to purify ER membrane proteins from HeLa cells in the required quantity and purity (i.e., without contaminating mitochondria).

Based on the ATP and ADP transport characteristics of the heterologously expressed SLC35B1 isoforms 1 and 2 and their similarities to the ATP and ADP transport activities that are present in proteoliposomes with the full complement of mammalian ER membrane proteins, the two SLC35B1 isoforms 1 and 2 are good candidates to work as ATP/ADP exchangers in the human ER membrane.

Depletion of SLC35B1 from HeLa cells reduces ER ATP levels. To directly address whether SLC35B1 acts as a transporter of chemical energy in the ER in human cells, HeLa cells were treated with two different $S L C 35 B 1$-targeting siRNAs for $96 \mathrm{~h}$, and the knockdown efficiencies were evaluated by quantitative RT-PCR (qRT-PCR) analysis. The analysis showed that mRNA depletion was efficient: the $5^{\prime}$ untranslated region (UTR)-targeting siRNA knocked down the residual SLC35B1 level to $\sim 10 \%$ and the coding region-targeting siRNA to $\sim 20 \%$ (Fig. $5 \mathrm{a}$ ). Western blot analysis with the anti-SLC35B1 antibody indicated that the siRNAs cause efficient protein depletion in SLC35B1-GFP expressing cells (Supplementary Fig. 1c). However, the Western blot approach for endogeneous SLC35B1 failed, most likely due to the low level of native SLC35B1 in HeLa cells ${ }^{17}$ (see Supplementary Fig. 1e). Depletion of SLC35B1 for $96 \mathrm{~h}$ did not cause any major alterations in whole cell or ER morphology, but led to slight over-expression of the apoptosis inducer $\mathrm{CHOP}^{24}$ and to concomitant decelerated cell growth, which was more pronounced for the more efficient SLC35B1UTR siRNA (Fig. 5b, c).

Next, the energy status of SLC35B1-depleted cells was characterized with time-resolved live cell recordings of ER ATP levels using the ER-targeted, genetically encoded ATP FRET sensor ERAT4.0125 (Fig. 6a). ATP levels were detected and compared in the ER of HeLa cells treated either with two different SLC35B1-targeting or control siRNAs, followed by ERAT4.01 transfection. SLC35B1 knockdown was correlated with significantly lower ATP levels in the ER compared to control cells (Fig. 6b, phase 1; quantification in Fig. 6c). Thapsigargin (Tg), which inhibits sarcoplasmic/endoplasmic reticulum $\mathrm{Ca}^{2+}$ ATPase (SERCA) in the ER membrane and stimulates $\mathrm{Ca}^{2+}$ release from the ER, led to the expected increase in the ER ATP levels ${ }^{25}$ (Fig. 6b, phase 2; quantification in Fig. 6d). The succeeding application of 2-deoxy-glucose (2-DG), which reduces the availability of ATP in the cytosol due to inhibition of glycolysis, induced the expected drop in ER ATP levels ${ }^{25}$ (Figs. 6b and 7a). In contrast, the response of $S L C 35 B 1$ knockdown cells to both $\mathrm{Tg}$ and 2-DG was less pronounced (Figs. 6b, d and 7a). Thus, SLC35B1 knockdown in HeLa cells reduced ATP levels in the ER and these levels could not be replenished by Tg-induced $\mathrm{Ca}^{2+}$ efflux from the ER.

To further substantiate the observed siRNA effects, we performed complementation analyses. Specifically, the ATP imaging experiments that were conducted in the presence of the SLC35B1-UTR-targeting siRNA were repeated in the presence of SLC35B1-expression or SLC35B1/Isoform 2-expression plasmids that lacked the UTR. Under these complementation conditions, the ATP levels in the ER were rescued to the levels in the control conditions, and the rescued cells responded to $\mathrm{Tg}$ 
a

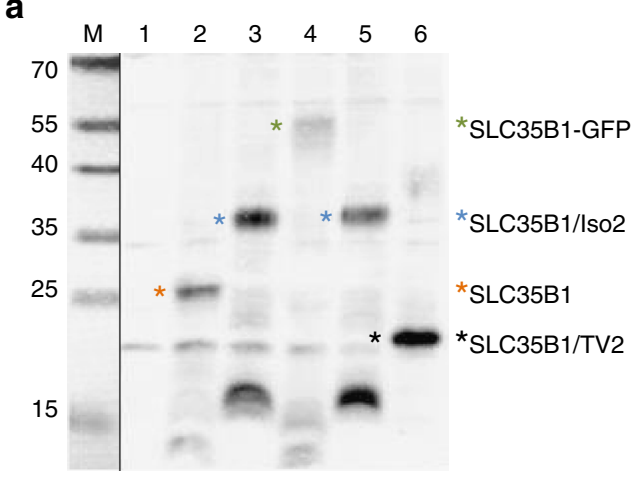

b

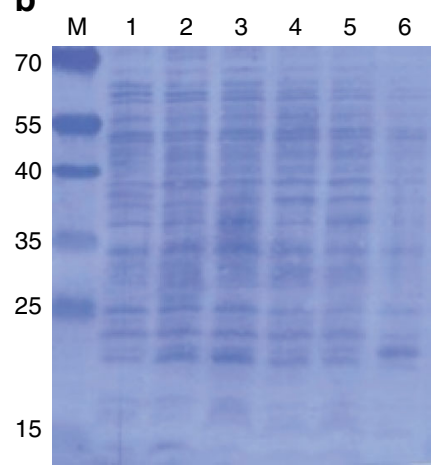

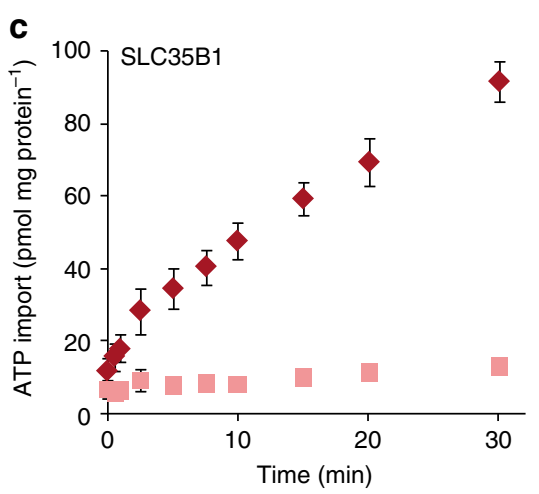
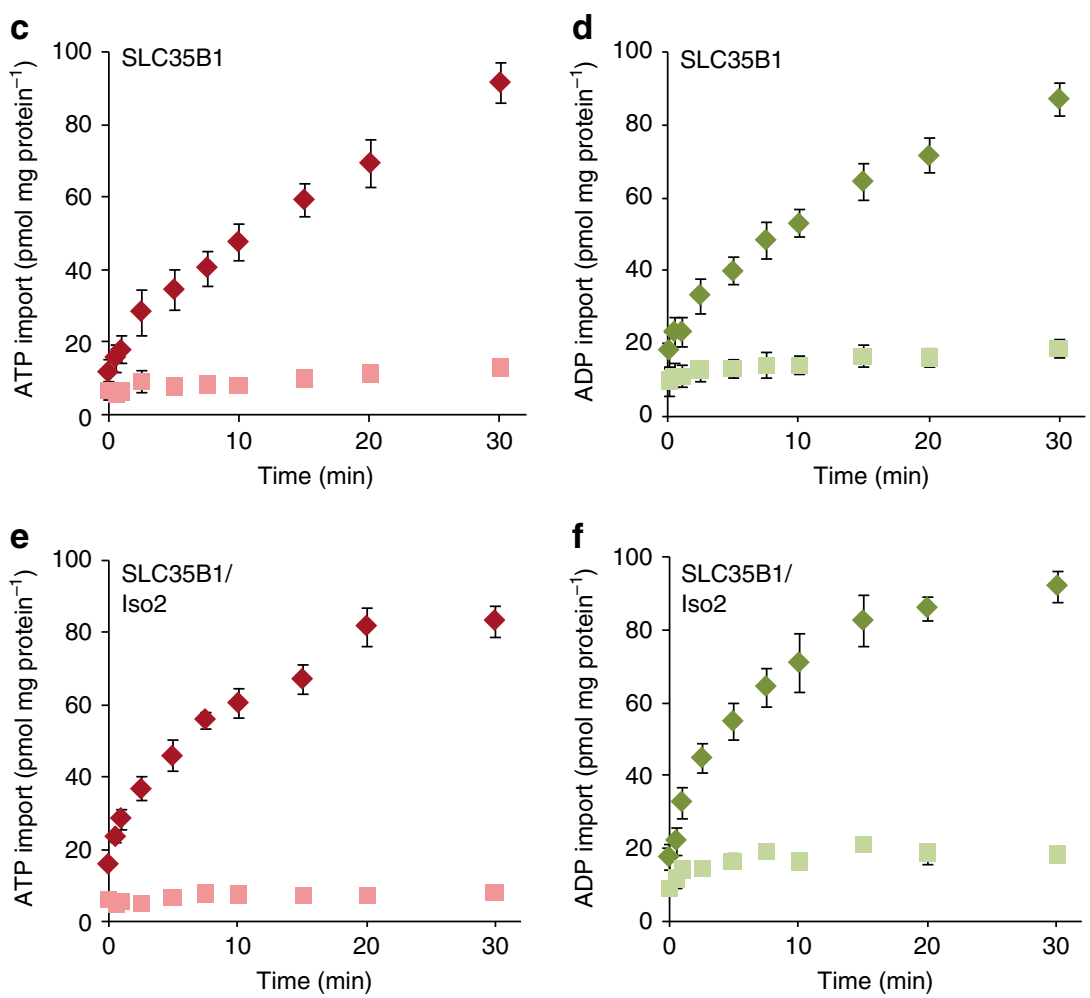

Fig. 2 Heterologously expressed SLC35B1 and SLC35B1/Isoform 2 are ATP and ADP carriers. The indicated SLC35B1 variants (labeled by asterisk in a, which were described in the legend to Fig. 1a, plus a phosphomimetic variant of Isoform 2 with aspartate residues at positions 15 and 29 instead of serines (termed Isoform 2DD, lane 5), and the GFP-tagged version of SLC35B1 were expressed in different E. coli cells. a, b Membranes were isolated from the indicated E. coli cells, and samples ( $25 \mu$ g protein) analyzed by SDS-PAGE and Western blotting using an SLC35B1-specific antibody, which was characterized in Supplementary Fig. 1a (a). Non-induced cells served as a negative control and are shown in lane 1. Coomassie staining the blot for total protein served as a loading control (b). A molecular mass standard $(M)$ was run in parallel and electronically copied from the stained blot to the Western blot image. c-f Uptake of $50 \mu \mathrm{M}\left[\alpha^{32}\right.$ P]ATP (c-e) or [ $\alpha^{32}$ P]ADP (d-f) into SLC35B1-expressing (c, d) or SLC35B1/Isoform 2-expressing cells (e, f) was measured and compared to uptake by non-induced cells. Data are reported as the mean of at least three independent experiments and are shown with the standard error of the mean (SEM). Related substrate saturation experiments and corresponding Eadie-Hofstee analyses revealed apparent $\mathrm{K}_{M}$ values for ATP and ADP plus maximal uptake rates and are shown in Supplementary Fig. 4

and 2-DG with the expected increase and decrease, respectively, in ATP levels in the ER, similar to the control cells (Fig. 7a-d).

In summary, the results from the heterologous expression experiments, SLC35B1 knockdown in HeLa cells in combination with live cell imaging of ATP levels in the ER, and complementation experiments indicated that SLC35B1 and SLC35B1/Isoform 2 represent the ATP/ADP exchangers in the human ER membrane that are responsible for net import of chemical energy into the ER.

Depletion of SLC35B1 from HeLa cells inhibits BiP activity. ER lumenal ATP allows BiP to fulfill its physiological roles, e.g., in facilitating selective protein import into the $\mathrm{ER}^{6,7,26}$ and in limiting $\mathrm{Ca}^{2+}$ leakage from the $\mathrm{ER}^{6,16}$, both by affecting gating of the Sec61 channel. Therefore, we expected reduced BiP activity as a result of SLC35B1 knockdown. This was first tested by analyzing $\mathrm{BiP}$-dependent protein import into the $\mathrm{ER}^{26}$. In these experiments, SLC35B1 was depleted from HeLa cells by treatment with two different SLC35B1-targeting siRNAs for $96 \mathrm{~h}$. Subsequently, the depleted cells were converted to semi-permeabilized cells and analyzed for their in vitro protein import capacity in parallel to semi-permeabilized cells from non-targeting siRNA-treated cells $^{26}$. For protein import analysis, established BiP-dependent (preproapelin) ${ }^{26}$ and BiP-independent precursor polypeptides 

Table 1 Impact of potential substrates and effectors on ATP
transport by SLC35B1

\begin{tabular}{|c|c|c|c|c|}
\hline \multirow[t]{2}{*}{ Effector } & \multicolumn{2}{|l|}{ SLC35B1 } & \multicolumn{2}{|c|}{ SLC35B1/1isoform 2} \\
\hline & Transport (\%) & $\begin{array}{l}\text { 土 SEM } \\
\text { (\%) }\end{array}$ & Transport (\%) & $\begin{array}{l}\text { \ SEM } \\
\text { (\%) }\end{array}$ \\
\hline ATP & 37.1 & 4.5 & 31.2 & 3.0 \\
\hline ADP & 48.6 & 3.1 & 43.5 & 3.7 \\
\hline AMP & 85.1 & 7.4 & 88.0 & 4.3 \\
\hline UTP & 114.0 & 12.6 & 106.4 & 12.9 \\
\hline GTP & 124.2 & 16.5 & 100.1 & 11.9 \\
\hline CTP & 122.6 & 10.2 & 109.2 & 7.7 \\
\hline UDP glucose & 129.5 & 13.5 & 105.9 & 8.4 \\
\hline $\begin{array}{l}\text { UDP } \\
\text { galactose }\end{array}$ & 107.3 & 5.6 & 93.9 & 1.5 \\
\hline UMP & 105.4 & 14.6 & 98.1 & 3.9 \\
\hline UDP & 126.8 & 19.5 & 92.7 & 2.9 \\
\hline $\begin{array}{l}\text { UDP-Nac- } \\
\text { Glc-AmN }\end{array}$ & 120.5 & 13.9 & 106.5 & 12.3 \\
\hline $\begin{array}{l}\text { GDP } \\
\text { mannose }\end{array}$ & 110.4 & 3.7 & 99.5 & 7.9 \\
\hline $\begin{array}{l}\mathrm{Mg}^{2+} 500 \\
\mu \mathrm{M}\end{array}$ & 113.4 & 9.6 & 106.5 & 5.2 \\
\hline $\mathrm{Ca}^{2+} 10 \mu \mathrm{M}$ & 105.5 & 7.6 & 106.7 & 12.5 \\
\hline $\mathrm{Ca}^{2+} 50 \mu \mathrm{M}$ & 119.8 & 19.0 & 109.6 & 7.0 \\
\hline $\begin{array}{l}\text { EDTA } 200 \\
\mu \mathrm{M}\end{array}$ & 146.8 & 10.8 & 114.0 & 10.8 \\
\hline EGTA $20 \mu \mathrm{M}$ & 114.6 & 4.9 & 103.6 & 6.2 \\
\hline $\begin{array}{l}\text { EGTA } 200 \\
\mu \mathrm{M}\end{array}$ & 120.8 & 9.2 & 105.3 & 8.6 \\
\hline
\end{tabular}

Note: Cellular uptake of $50 \mu \mathrm{M}$ of [ $\left.\alpha^{32} \mathrm{P}\right] \mathrm{ATP}$ by recombinant SLC35B1 isoforms proceeded for 5 min (considered 100\%). The corresponding transport in the presence of selected potential nonlabeled substrates $(500 \mu \mathrm{M})$ or effectors was calculated accordingly. Rates are reported as the mean of at least three independent experiments and represent net values (minus ATP import by non-induced cells). Standard errors of the mean are given $( \pm S E M)$. Bold values indicate significantly reduced transport $(<60 \%$ of control)

$(\operatorname{Sec} 61 ß)^{26}$ were synthesized and radiolabeled in rabbit reticulocyte lysate and import efficiency was evaluated via SDS-PAGE and phosphorimaging. BiP-dependent protein import was found to be reduced by SLC35B1 knockdown, whereas BiP-independent transport was unaffected (Fig. 8a-d). Thus, SLC35B1 depletion phenocopied the effect of BiP depletion on BiP-dependent ER protein import ${ }^{26}$.

In a second approach, HeLa cells were treated with the two different SLC35B1-targeting siRNAs for $96 \mathrm{~h}$ and loaded with the ratiometric $\mathrm{Ca}^{2+}$ indicator Fura- $2^{6,27}$, to address by live cell $\mathrm{Ca}^{2+}$ imaging if SLC35B1 depletion also affects $\mathrm{BiP}^{\prime}$ s role in limiting $\mathrm{Ca}^{2+}$ leakage from the $\mathrm{ER}^{6,16}$. SLC35B1 knockdown by both siRNAs decreased the $\mathrm{Ca}^{2+}$ concentration in the ER lumen, which was measured as Tg-releasable $\mathrm{Ca}^{2+}$ (Fig. 8e-g; statistics in Fig. 8f-h), and it also decreased total Ionomycin-releasable $\mathrm{Ca}^{2+}$ (Fig. 8i-k; statistics in Fig. 8j-1). Thus, ATP depletion in the ER driven by SLC35B1 knockdown decreased $\mathrm{ER} \mathrm{Ca}^{2+}$, i.e., stimulated ER $\mathrm{Ca}^{2+}$ efflux, as it had previously been observed after BiP depletion ${ }^{6}$.

Two possibilities could in principle account for this result, decreased $\mathrm{Ca}^{2+}$ uptake or increased $\mathrm{Ca}^{2+}$ release from the ER. Considering the importance of the Sec61 protein translocon as a passive $\mathrm{ER} \mathrm{Ca}^{2+}$ release channel ${ }^{16,27,28}$, and the ER luminal chaperone $\mathrm{BiP}$ as its allosteric regulator ${ }^{6,29}$, we hypothesized that the observed drop in ATP levels in the ER lumen in response to SLC35B1 knockdown might activate $\mathrm{Ca}^{2+}$ leakage from the ER via open Sec61 channels. This hypothesis was tested by arresting precursor polypeptides in transit through the Sec61 translocon, i.e., blocking the passive $\mathrm{Ca}^{2+}$ leak channel, using the elongation inhibitor emetine along with SLC35B1 knockdown and live cell
$\mathrm{Ca}^{2+}$ imaging ${ }^{28}$. Emetine rescued $\mathrm{Ca}^{2+}$ homeostasis after SLC35B1 knockdown with both siRNAs by limiting $\mathrm{Ca}^{2+}$ efflux from the ER via Sec61 channels (Fig. 8i-k; statistics in Fig. 8j-l).

Thus, depletion of ATP from the ER results in reduced $\mathrm{BiP}$ activity, which causes reduced $\mathrm{BiP}$-dependent ER protein import and increased ER $\mathrm{Ca}^{2+}$ efflux. Therefore, these results confirm the conclusion that SLC35B1 represents the ATP/ADP exchanger in the human ER membrane.

SLC35B1 may be part of a $\mathrm{Ca}^{2+}$-dependent regulatory circuit. Finally, we tested if SLC35B1 is involved in controling cellular energy homeostasis in response to ER ATP depletion. AMPK is the master regulator of energy metabolism ${ }^{25,30-32}$ and can be activated by a decrease in cytosolic ATP levels or by an increase in cytosolic $\mathrm{Ca}^{2+}$ via the calcium/calmodulin dependent kinase kinase $2(\mathrm{CAMKK} 2)^{31}$.

First, we addressed cytosolic ATP levels as the potential AMPK regulator. Therefore, we monitored ATP levels in the cytosol in real-time using the cytosolic ATP sensor ATeam ${ }^{33}$ and in cellular lysates using a bioluminescent assay. SLC35B1 depletion from HeLa cells did not significantly affect cytosolic ATP levels or the total cellular ATP levels (Fig. 6e-g), which is consistent with the facts that HeLa cells are not professional secretory cells and that the ER in HeLa cells does not significantly contribute to the total cellular ATP levels.

The results shown in Figs. 6 and 7 already suggested that Tginduced increase in cytosolic $\mathrm{Ca}^{2+}$ and the concomitant increase in ATP levels in the ER were an effect of $\mathrm{Ca}^{2+}$ on cytosolic ATP production. Therefore, we hypothesized that the observed drop in ATP levels in the ER lumen in response to SLC35B1 knockdown might activate a signaling mechanism that controls ER and cytosolic $\mathrm{Ca}^{2+}$ levels. This cascade would start with reduction in $\mathrm{BiP}$ activity and subsequent increase in $\mathrm{Ca}^{2+}$ leakage from the ER via open Sec61 channels. This hypothesis was tested positive in the above-described $\mathrm{Ca}^{2+}$ imaging experiments. In brief, SLC35B1 knockdown decreased the $\mathrm{Ca}^{2+}$ concentration in the ER lumen (Fig. 8e-l) and emetine rescued $\mathrm{Ca}^{2+}$ homeostasis after SLC35B1 knockdown by limiting $\mathrm{Ca}^{2+}$ efflux from the ER via Sec61 channels (Fig. 8j-1). These results were consistent with previous work that showed that $\mathrm{Ca}^{2+}$ efflux from the ER into the cytosol acts as a signal that increases ADP phosphorylation in the cytosol $^{25}$ and expanded the model by adding three additional players, SLC35B1, BiP, and the Sec61 channel (Fig. 9).

At last, we asked how SLC35B1 knockdown may directly affect cytosolic ATP production. HeLa cells were treated with the two SLC35B1-targeting siRNAs for $96 \mathrm{~h}$ and the cell lysates were analyzed by Western blotting using antibodies against the phosphorylated alpha and beta subunits of AMPK. We found that SLC35B1 knockdown resulted in pronounced AMPK phosphorylation (Fig. $8 \mathrm{~m}, \mathrm{n}$ ), which is expected to stimulate cytosolic ATP production ${ }^{25}$.

\section{Discussion}

Taking together that SLC35B1 has ATP/ADP exchange acitivity similar to the transport activity that is present in pancreatic ER membranes and may be located in the ER membrane in canine pancreas and human cells, we conclude that SLC35B1 is likely the ATP/ADP exchanger in the ER membrane and suggest to name it AXER. Our findings explain why AXER orthologs in Saccharomyes cerevisiae (HUT1), Schizosaccharomyces pombe (HUT1), and Caenorhabditis elegans (HUT-1) have been found to play a more general role in ER homeostasis, as would be expected for a nucleotide sugar transporter ${ }^{12,13}$

AXER/Isoform 2 appears to be the only of the three known isoforms present in canine pancreatic microsomes. Which 
a
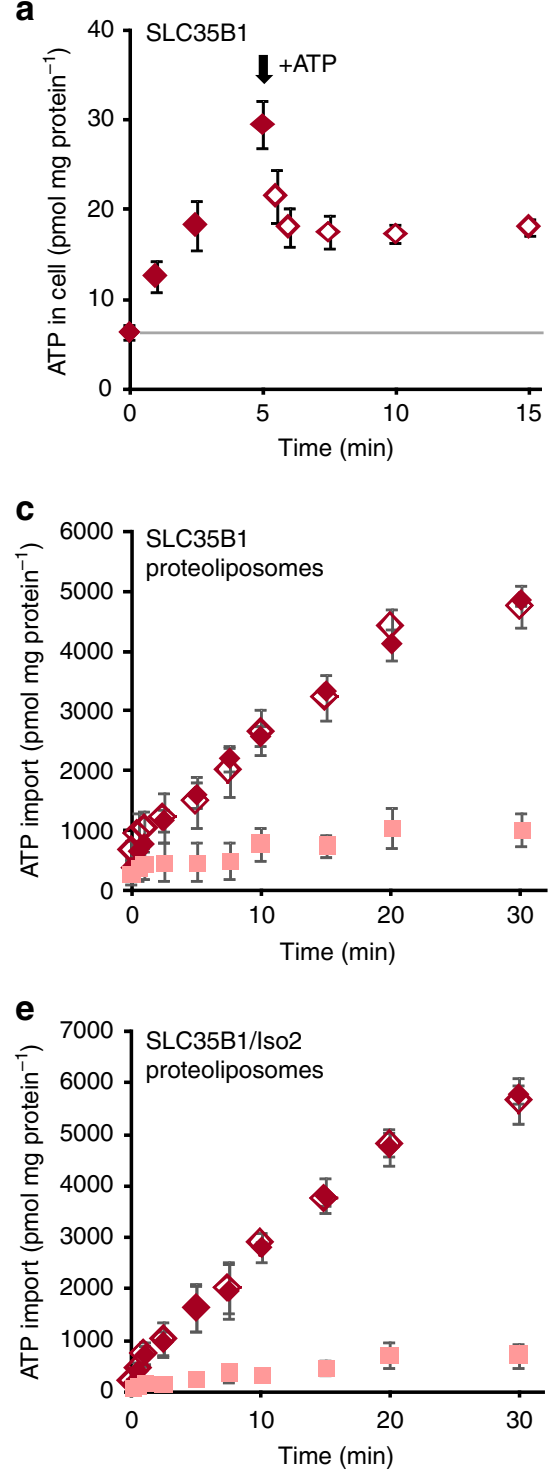

b

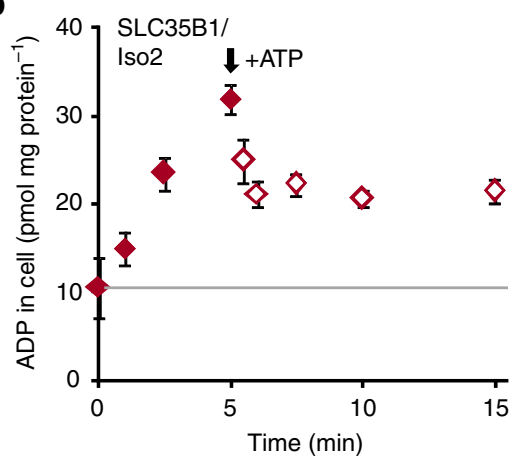

d
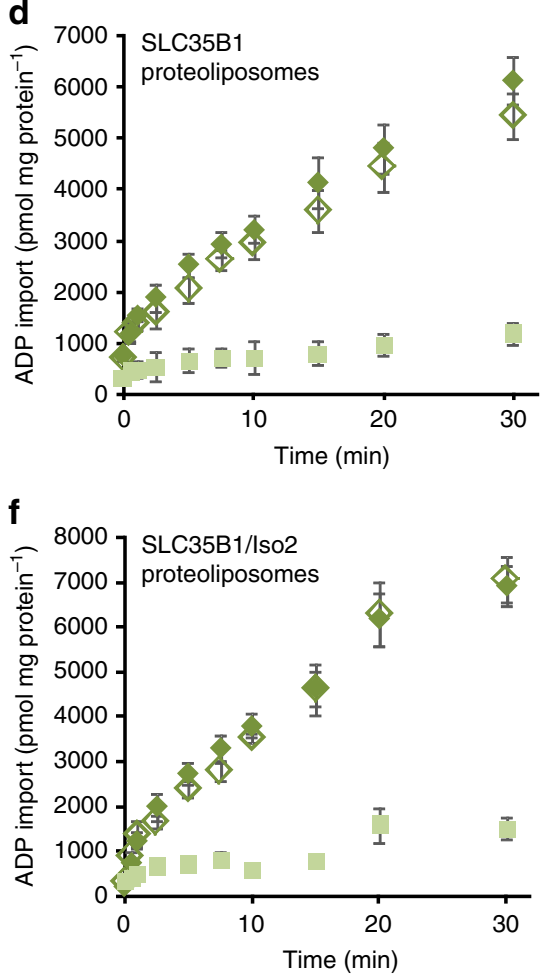

Fig. 3 Heterologously expressed SLC35B1 and SLC35B1/Isoform 2 are antiporters for ADP and ATP. a, b After 5 min of uptake of $20 \mu M$ [ $\alpha^{32}$ P]ATP as described in Fig. 2, efflux was induced by the addition of ten-fold molar excess of unlabeled ATP. Data are reported as the mean of at least three independent experiments and are shown with the standard error of the mean (SEM). See Supplementary Fig. 2 for the demonstration of ATP export into the cell supernatants under similar conditions. c-f Proteoliposomes harboring membrane proteins from SLC35B1 (c, d) or SLC35B1/Isoform 2 (e, f) transfected E. coli cells were prepared as described in Methods. Time dependent accumulation of $50 \mu \mathrm{M}\left[\alpha^{32} \mathrm{P}\right]$-ATP in unloaded (pink) or preloaded proteoliposomes (c, e) or $50 \mu \mathrm{M}\left[\alpha^{32} \mathrm{P}\right]$-ADP in unloaded (light green) or preloaded proteoliposomes (d, f). Proteoliposomes were preloaded with $10 \mathrm{mM}$ ADP (empty rhombs) or $10 \mathrm{mM}$ ATP (filled rhombs). [ $\left.\alpha^{32} \mathrm{P}\right]-$ ATP or [ $\left.\alpha^{32} \mathrm{P}\right]-$ ADP was present at a concentration of $50 \mu \mathrm{M}$. Uptake was terminated after $2 \mathrm{~min}$. Data are reported as the mean of at least three independent experiments and are shown with the standard error of the mean (SEM)

isoform(s) is (are) present in HeLa cells is not completely resolved. We note however that the agarose gel electrophoretic analysis of the PCR products from HeLa cells showed that there are mRNA molecules which are long enough to allow synthesis of Isoforms 1 and 2 (Supplementary Fig. 6), which is consistent with the presence of transcript variants in NCBI. According to UniProtKB serine residues at positions 15 and 29 of AXER/ Isoform 2 may be subject to phosphorylation. Therefore, we generated a phosphomimetic variant of AXER/Isoform 2 and characterized its carrier activity after heterologous expression in E. coli. We did not detect any effect of the two point mutations under these conditions, i.e., in the absence of potential cytsosolic interaction partners (Supplementary Fig. $3 c, d)$. Thus the phosphorylation per se does not seem to affect carrier activity.
According to a hypothetical structural model of the human AXER (Fig. 1b, Suppplementary Fig. 7a, b), as predicted by the Phyre2 server ${ }^{34}$, AXER can be expected to catalyze the equimolar exchange of adenosine di-phosphates and triphosphates by an alternating access mechanism, in which a single substrate binding site is made available either to the cytosolic ER surface or the ER lumen through conformational changes, which is reminiscent of the mitochondrial ADP/ATP carriers ${ }^{35}$. When AXER is modeled on the X-ray structure of the $S n Y d d g$ transporter ${ }^{15}$, two positive clusters that are flanking transmembrane domain 5 on the cytosolic and ER lumenal sides (Fig. 1a, b, Supplementary Fig. 7) suggest another analogy to the mitochondrial carriers, whereby the positive clusters are involved in both steering the substrates to the central cavity and stripping $\mathrm{Mg}^{2+}$ from the nucleotides, respectively. Notably, a stripping of $\mathrm{Mg}^{2+}$ from the nucleotides is 

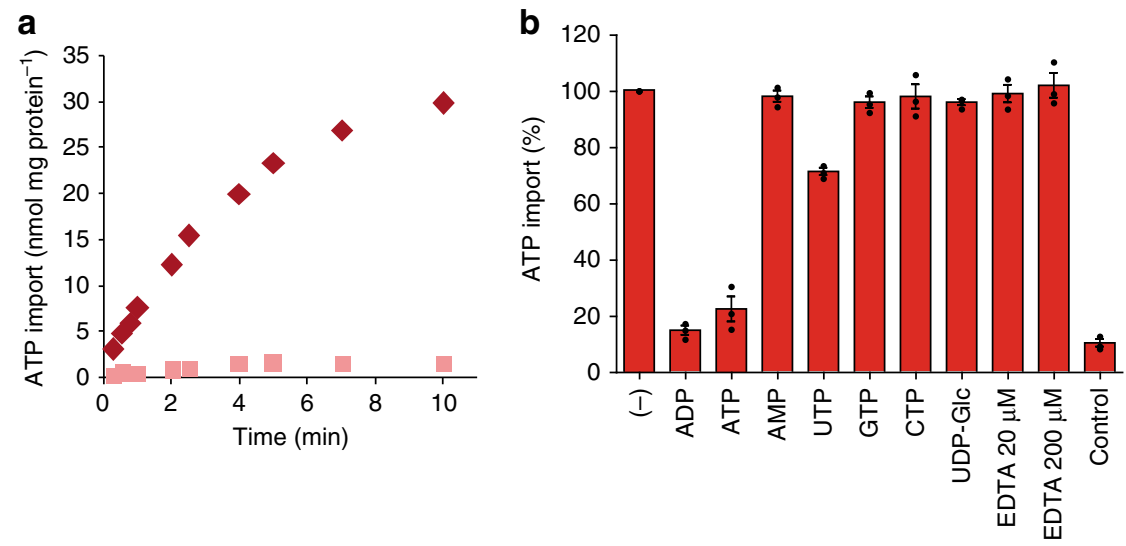

Fig. 4 ATP transport into proteoliposomes with the full complement of mammalian ER membrane proteins. Proteoliposomes harboring porcine or canine pancreatic rough ER membrane proteins were prepared as described in Methods. a Time dependent accumulation of $50 \mu M$ [ $\left.\alpha^{32} \mathrm{P}\right]-A T P$ in unloaded (pink) or preloaded (10 mM ADP; red) proteoliposomes, which were derived from porcine pancreatic microsomes by treaytment with puromycin and high salt (PKRM). Related substrate saturation experiments and corresponding Eadie-Hofstee analyses are shown in Supplementary Fig. 5. All data are reported as the mean of at least three independent experiments. b Proteoliposomes were preloaded with $10 \mathrm{mM}$ ADP (except for the bar labeled control, which refers to unloaded proteoliposomes). [ $\left.\alpha^{32} \mathrm{P}\right]$-ATP was present at a concentration of $50 \mu \mathrm{M}$. Each effector substance was given at a concentration of $500 \mu \mathrm{M}$ and uptake was terminated after $2 \mathrm{~min}$. All data are reported as the mean of at least three independent experiments and are shown with the standard error of the mean (SEM)
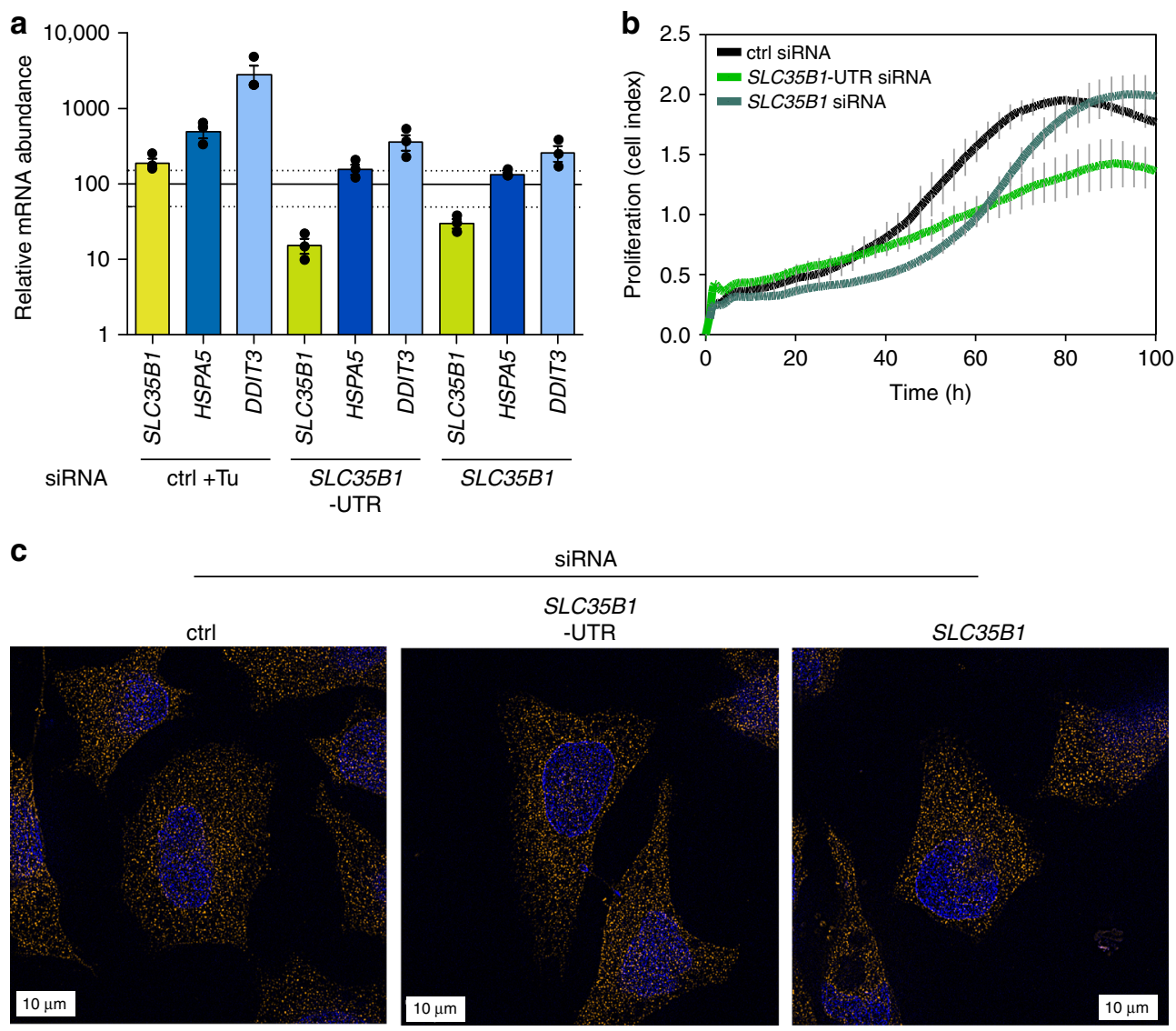

Fig. 5 SLC35B1 knockdown in HeLa cells does not dramatically affect cell growth, cell and ER morphology. a Quantitative RT-PCR performed after the transfection of HeLa cells with control siRNA or with SLC35B1- or SLC35B1-UTR targeting siRNA for $96 \mathrm{~h}$. One set of control siRNA samples was treated with tunicamycin $(\mathrm{Tu}, 2.5 \mu \mathrm{g} / \mathrm{ml})$ for $2 \mathrm{~h}$ in order to induce UPR ${ }^{40}$. Data from three independent experiments are reported together with the individual data points as $\%$ of control with SEM. The light lines indicate the 50 and $150 \%$ values. The HSPA5 gene encodes BiP, the DDIT3 gene CHOP. b HeLa cells were transfected with control siRNA or with SLC35B1-targeting or SLC35B1-UTR-targeting siRNA for $48 \mathrm{~h}$, transferred to $\times$ CELLigence plates $\left(5 \times 10^{3}\right.$ cells per well), and cultivated for $100 \mathrm{~h}$. Cell growth was monitored in real-time. c HeLa cells were transfected with control siRNA or with SLC35B1-UTR-targeting siRNA for $96 \mathrm{~h}$, stained with DAPI and with a Sec62-specific antibody, and analyzed on the super-resolution microscope ${ }^{38}$. Representative images are shown (scale bar $10 \mu \mathrm{m}$ ) 
a
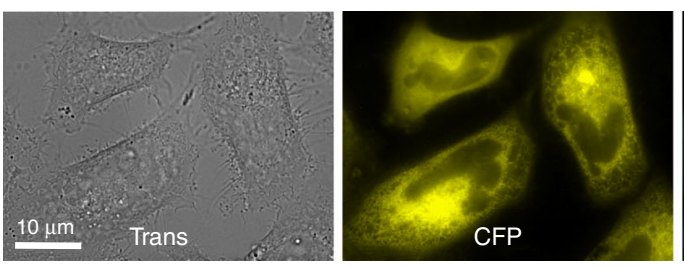

b

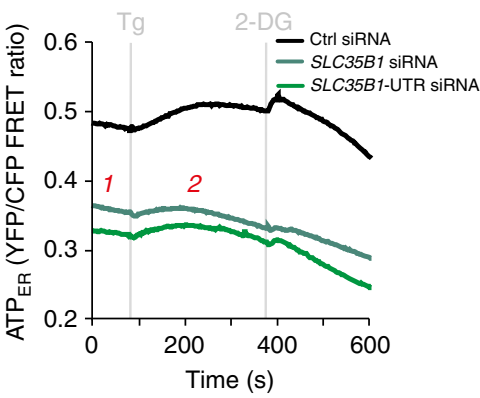

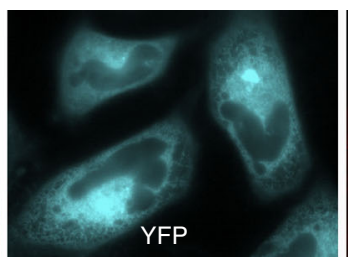
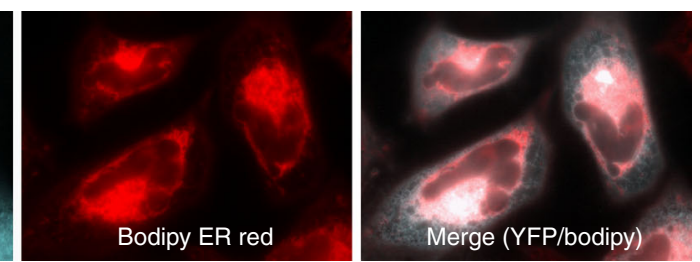

d

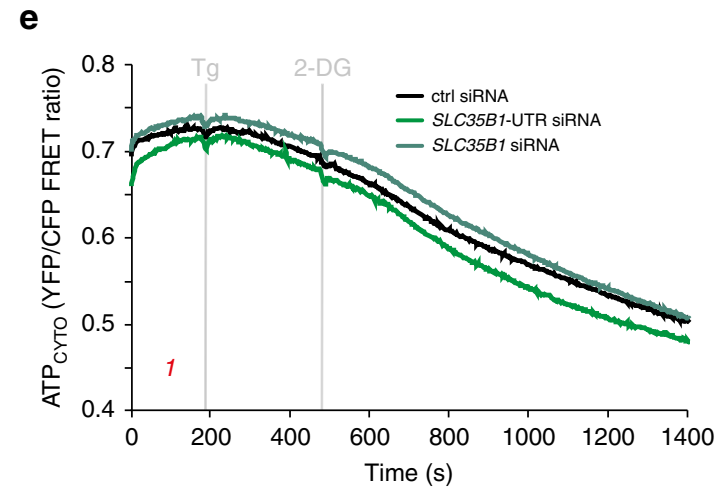

C

Resting $\operatorname{ATP}_{\mathrm{ER}}(1)$

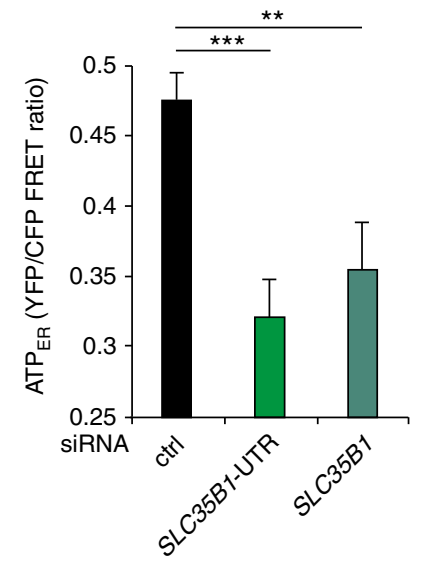

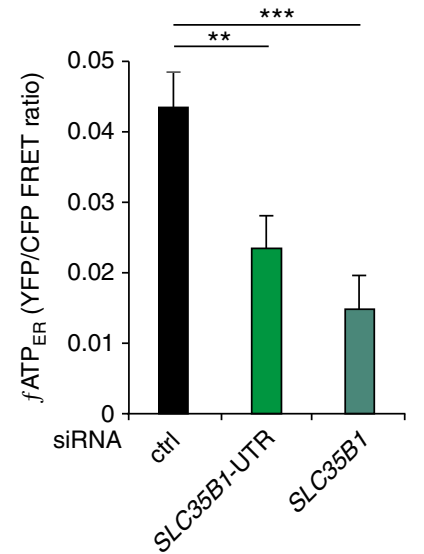

f

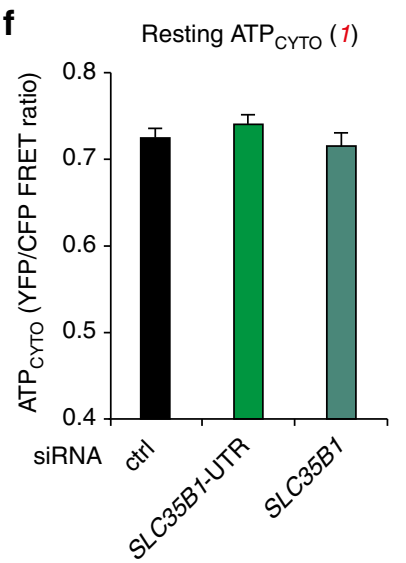

9

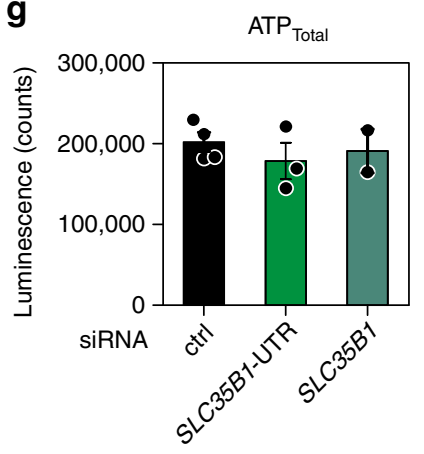

Fig. 6 ERAT4.01 reveals ATP depletion in the ER of HeLa cells after SLC35B1 knockdown. a-d HeLa cells were transfected with control siRNA or with SLC35B1- or SLC35B1-UTR-targeting siRNA for $72 \mathrm{~h}$, then transfected with ERAT4.01. After $24 \mathrm{~h}$, they were imaged by fluorescence microscopy. Where indicated, Thapsigargin $(\mathrm{Tg}, 1 \mu \mathrm{M})$ or 2-deoxy-glucose (2-DG, $10 \mathrm{mM})$ were added. a Images were recorded using transmission light or fluorescence. Representative images are shown (scale bar $10 \mu \mathrm{m}$ ). b Time-resolved live cell recordings of ER luminal ATP levels are shown as FRET-ratio $F_{535} / F_{480}$. Data are presented as means for ctrl, $n=33$ cells, UTR siRNA, $n=20$, SLC35B1 siRNA, $n=21$, from at least three independent experiments. c Statistical analysis of the resting ATP levels in the experiments shown in $\mathbf{b}$. Three time points before Tg addition were averaged (indicated as 1 ) and subtracted from the MAX-values (indicated as 2) following Tg addition for each single cell. Data are presented as mean with SEM. The indicated pairs were assessed by unpaired, two-sided standard Student's $t$-test $\left({ }^{\star} P<0.05,{ }^{\star \star} P<0.01,{ }^{\star \star} P<0.001\right)$. d Statistical analysis of the Tg-induced ATP increase in the experiments shown in $\mathbf{b}$. e, $\mathbf{f}$ HeLa cells were transfected with control siRNA or with SLC35B1- or SLC35B1-UTR-targeting siRNA, transfected with ATeam, and imaged. The mean values of time-resolved live cell recordings of cytosolic ATP levels (e) and the corresponding statistical analysis $(\mathbf{f})$ are shown for ctrl, $n=116$ cells, UTR siRNA, $n=95$, SLC35B1 siRNA, $n=57$ from at least three independent experiments. $\mathbf{g}$ HeLa cells were transfected with control siRNA or with SLC35B1-targeting or SLC35B1-UTR-targeting siRNA for $96 \mathrm{~h}$, and total cellular ATP was determined using ApoSensor according to the manufacturer's protocol. Data from three independent experiments are reported together with the individual data points as \% of control with SEM

consistent with the observed EDTA insensitivity of both heterologously expressed SLC35B1 and the carrier, which is present in proteoliposomes, harboring the full complement of mammalian ER membrane proteins (Table 1, Fig. 4b). Furthermore, transport direction can be expected to be directed by the ATP gradient.

In human cells, AXER appears to be part of a regulatory circuit and a $\mathrm{Ca}^{2+}$-dependent signaling pathway, termed lowER, acting in vicinity of the ER and guaranteeing sufficient ATP supply to the ER (Fig. 9). The initial experimental data to characterize this putative signaling pathway, which were presented here, suggest the following scenario for lowER: High ATP/ADP ratio in the ER allows $\mathrm{BiP}$ to limit $\mathrm{Ca}^{2+}$ leakage from the ER via the Sec61 channel $^{6}$. Low ATP/ADP ratio due to increased protein import and folding or due to protein misfolding, leads to $\mathrm{BiP}$ dissociation from the Sec61 channel thus inducing $\mathrm{Ca}^{2+}$ leakage from the $\mathrm{ER}^{6}$. In the cytosol, $\mathrm{Ca}^{2+}$ binds to calmodulin (CaM) near the ER 
a

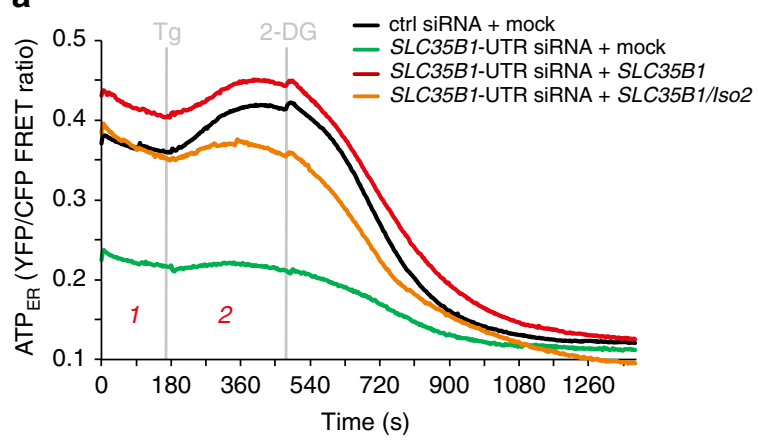

b

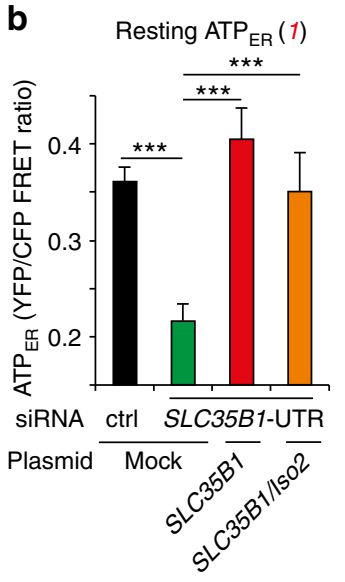

C

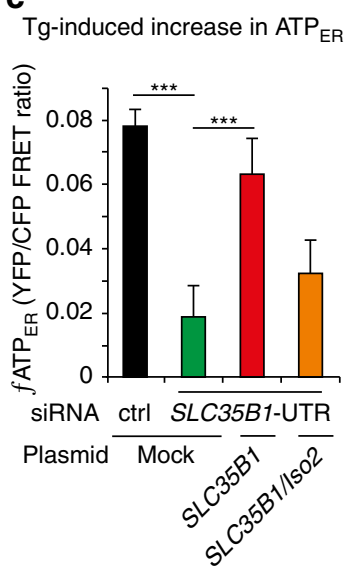

d

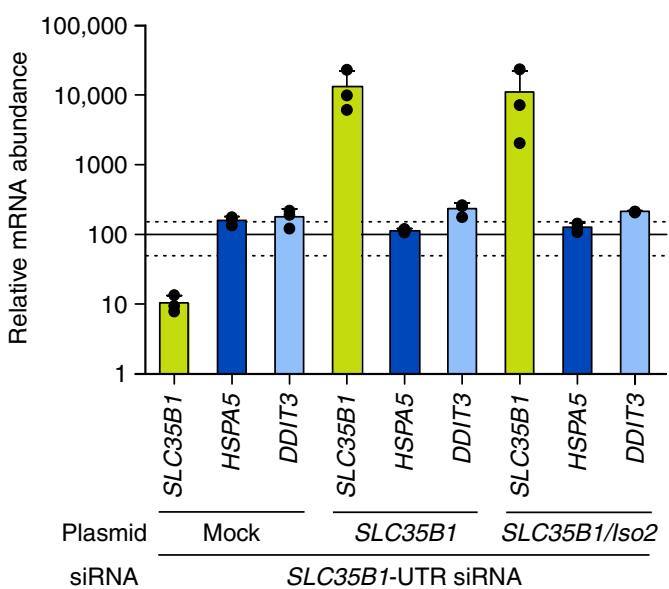

Fig. 7 SLC35B1 expression rescues ATP levels in the ER after SLC35B1 knockdown. a-c HeLa cells were transfected with control siRNA or with SLC35B1-UTRtargeting siRNA plus SLC35B1 expression plasmids for $72 \mathrm{~h}$. The cells were transfected with ERAT4.01. After $24 \mathrm{~h}$, they were imaged by fluorescence microscopy, and the FRET-ratio $\mathrm{F}_{535} / \mathrm{F}_{480}$ was recorded in real-time. Where indicated, $1 \mu \mathrm{M} \mathrm{Tg}$ or $10 \mathrm{mM}$ 2-DG were added. a Time-resolved live cell recordings of ER luminal ATP levels. Data are presented as means of ctrl, $n=71$ cells, UTR siRNA, $n=39$, SLC rescue, $n=18$, and Iso2 rescue, $n=13$ from at least three independent experiments. $\mathbf{b}$ Quantification of resting ATP ER concentration in $\mathbf{a}$. $\mathbf{c}$ Quantification of Tg-induced ATP ER concentration in a. Three time points before Tg addition were averaged (indicated as 1) and subtracted from the MAX-values (indicated as 2) following Tg addition for each single cell. Data in $\mathbf{b}$ and $\mathbf{c}$ are presented as mean with SEM. The indicated pairs were assessed by unpaired, two-sided standard Student's $t$-test $\left({ }^{\star} P<0.05,{ }^{\star \star} P<0.01,{ }^{\star \star} P<0.001\right)$. d Mean quantitative RT-PCR values are shown after transfection of HeLa cells with control siRNA or with SLC35B1-UTR targeting siRNA plus SLC35B1 expression plasmids. Data from three independent experiments are reported together with the individual data points as \% of control with SEM. The light lines indicate the 50 and 150\% values. The HSPA5 gene encodes BiP, the DDIT3 gene CHOP

surface $^{27}$, and activates AMPK via CAMKK2 and finally 6phospho-fructo-2-kinase $(\mathrm{PF} 2 \mathrm{~K})^{25}$. Activated $\mathrm{PF} 2 \mathrm{~K}$ causes increased ADP phosphorylation in glycolysis, leading to ATP import into the ER via AXER, which is also activated by $\mathrm{Ca}^{2+}$ efflux from the $\mathrm{ER}^{25}$. Interestingly, mammalian AXER comprises an IQ motif in the cytosolic loop between transmembrane domains 2 and 3 (Fig. 1a, b) and, thus, may also be activated by $\mathrm{Ca}^{2+}$-CaM (Supplementary Fig. 8). Normalization of the ER $\mathrm{ATP} / \mathrm{ADP}$ ratio, causes $\mathrm{BiP}$ to limit the $\mathrm{Ca}^{2+}$ leakage and thus inactivates the signal transduction pathway. SERCA, which pumps $\mathrm{Ca}^{2+}$ back into the ER lumen, balances the passive $\mathrm{Ca}^{2+}$ efflux and protein phosphatase 2 (PP2) dephosphorylates AMPK. Note that (i) all mentioned proteins are present in sufficient quantities in the HeLa cells which were used here (Supplementary Table 1); (ii) it is expected that the lowER involves sites of contact between ER and mitochondria and oxidative phosphorylation as an energy source in non-cancer cells; and (iii) activated AMPK was shown previously to lead to reduced cap-dependent translation and therefore ties the lowER to the UPR ${ }^{30}$. While ADP is exported via AXER, phosphate may leave the ER via the Sec61 channel, which is not ion selective ${ }^{16}$ and is also permeable to glutathione $\mathrm{e}^{36}$.
Our data suggest that AXER is not only an ATP importer and ADP exporter in the ER membrane, but may also be part of an ER to cytosol low energy response regulatory axis (termed lowER). It remains open if AXER is the only ATP carrier in the ER membrane of mammalian cells, its ortholog in C. elegans is essential only during larval development but not in the adult worm ${ }^{13}$. The proposed $\mathrm{Ca}^{2+}$-dependent regulatory circuit low ER guarantees ER energy metabolism and ER proteostasis under physiological conditions and clearly awaits further validation and characterization. Under non-physiological conditions, it can be expected to represent the first line of defense of a cell against ER stress and, therefore, may interact with the unfolded protein response $\mathrm{e}^{30}$.

\section{Methods}

Materials. Expression plasmids for carboxyterminally Myc-DDK-tagged SLC35B1 (RC204145), GFP-tagged SLC35B1 (RG204145), Myc-DDK tagged transcript variant 2 (RC236977), and Myc-DDK-tagged SLC35B1/Isoform2 (RC204145, customized), as well as transient over-expression lysate of SLC35B1 (LY401770) were from OriGene, as was anti-Myc-DDK antibody (TA50011, mouse monoclonal, used dilution: 1:1,000). The expression plasmid for ATP sensor ERAT4.01 was purchased from Next Generation Fluorescence Imaging. ANTI-FLAG M2 affinity gel (A2220), antibodies against $\beta$-actin (A5441, mouse monoclonal, used dilution: 1:10,000) and SLC35B1 (HPA057418, rabbit polyclonal, affinity purified, 
a

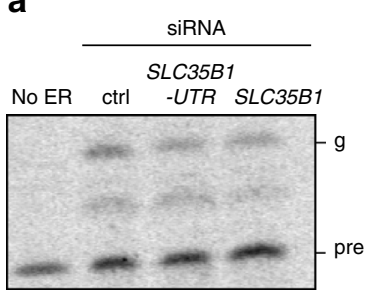

e

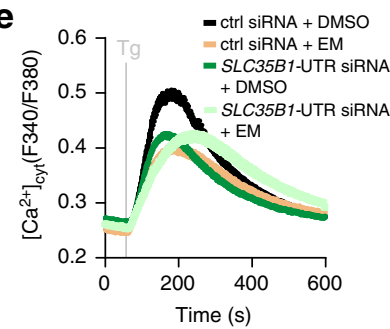

f

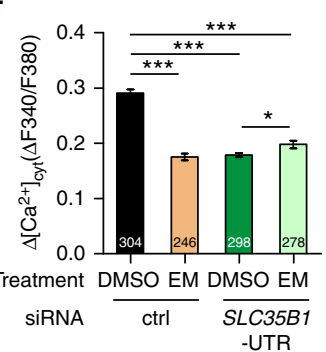

b

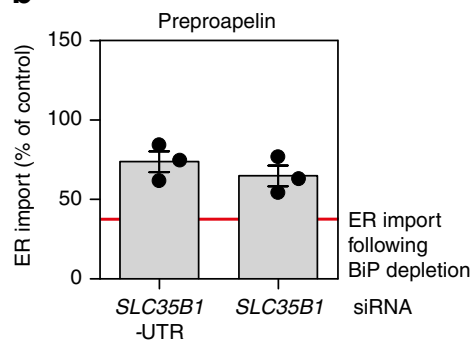

g

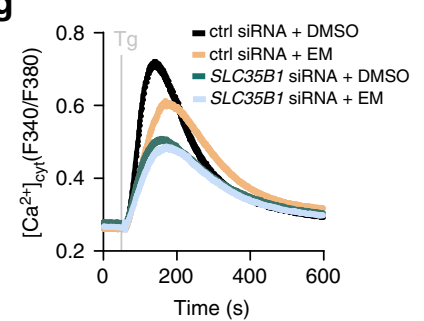

h

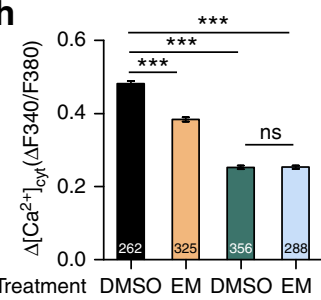

Treatment DMSO EM DMSO EM
C

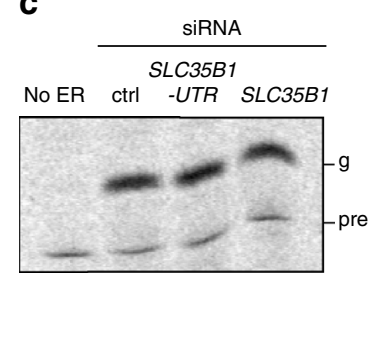

i

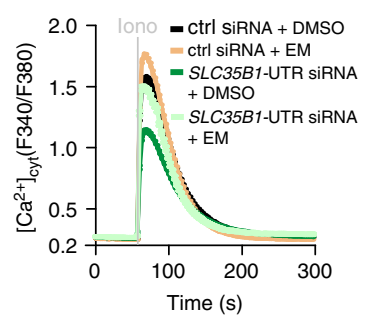

j

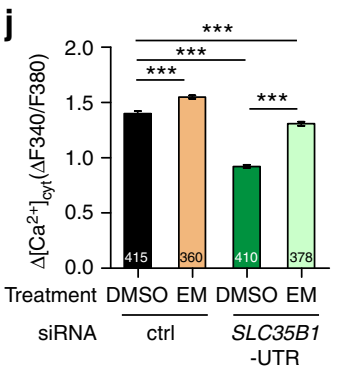

d

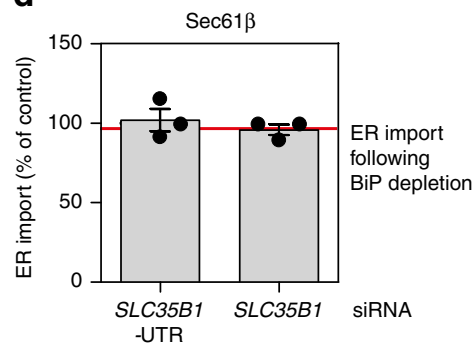

k

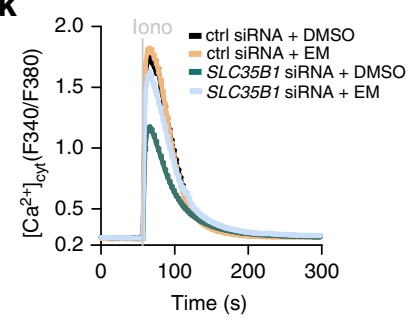

I

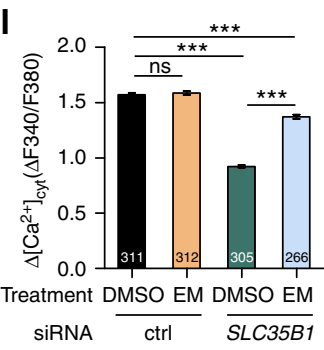

m
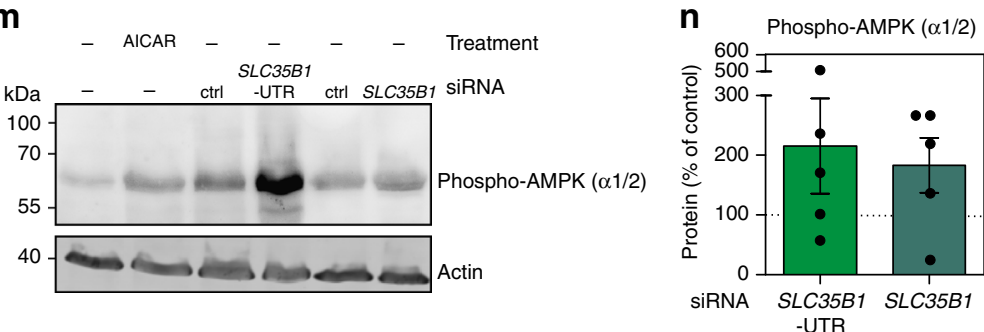

Fig. 8 Knockdown of SLC35B1 leads to inhibition of BiP activity and to AMPK phosphorylation. a-d HeLa cells were transfected with control siRNA or with SLC35B1-targeting or SLC35B1-UTR-targeting siRNA for $96 \mathrm{~h}$. They were converted to semi-permeabilized cells and tested for protein transport activity as described previously $6,26,38,39$. Here, ER protein import, assayed as $\mathrm{N}$-glycosylation, of the small presecretory protein preproapelin ${ }^{26}$ (which depends on $\mathrm{BiP}$, $\left(\mathbf{a}, \mathbf{b}\right.$ ), and of the tail anchored $\operatorname{Sec} 61 \mathrm{~B}^{26}$ (which does not depend on $\mathrm{BiP},(\mathbf{c}, \mathbf{d}$ ) were analyzed. The effects of siRNA-mediated depletion of BiP are shown for comparison ${ }^{26}$. Representative phosphorimages after SDS-PAGE and quantitative data from three independent experiments are reported together with the individual data points as \% of control with SEM. Pre, precursor form; g, glycosylated form. e-I HeLa cells were transfected with control siRNA or with SLC35B1- or SLC35B1-UTR-targeting siRNA for $96 \mathrm{~h}$. Where indicated, $0.0001 \%$ DMSO, or $10 \mu \mathrm{M}$ emetine (EM) were present during the last $2 \mathrm{~h}$ of growth plus Fura loading. Cells were loaded with Fura-2 for $30 \mathrm{~min}$, transferred to $\mathrm{Ca}^{2+}$-free buffer, and Fura-2 signals were recorded as $\mathrm{F}_{340} / \mathrm{F}_{380}$ ratios in realtime ${ }^{6,27}$. Where indicated, $1 \mu \mathrm{M}$ Thapsigargin $(\mathrm{Tg})$, or $5 \mu \mathrm{M}$ lonomycin (Iono) were added. $\mathbf{e}, \mathbf{g}, \mathbf{i}, \mathbf{k}$ The mean values of the ratiometric recordings are shown with the standard error of the mean (SEM). $\mathbf{f}, \mathbf{h}, \mathbf{j}, \mathbf{I}$ Statistical analysis of Tg-induced ( $\mathbf{f}-\mathbf{h})$ or lono-induced (j-I) changes in cytosolic Ca ${ }^{2+}$ levels in the experiments with the indicated number of cells in at least three independent experiments. The indicated pairs were assessed by unpaired, two-sided Student's $t$-test $\left({ }^{\star} P<0.05,{ }^{\star \star} P<0.01,{ }^{\star \star} P<0.001, \mathrm{~ns}\right.$, not significant). $\mathbf{m}, \mathbf{n}$ Where indicated, $0.5 \mathrm{mM}$ AICAR was present for $15 \mathrm{~h}$. Cells were treated as indicated and analyzed by SDS-PAGE and Western blotting using phosphorylated AMPK-specific antibodies. Notably, AICAR is an AMP-mimetic and AMPK activator ${ }^{46}$. Staining the blot for $B$-actin served as a loading control. Representative luminescence images of the blots and quantitative data from five independent experiments are reported together with the individual data points as \% of control with SEM

used dilution: 1:200) were obtained from Sigma, antibodies against tGFP (AB514, rabbit polyclonal, used dilution 1:2,500) were from Evrogen, and antibodies against phosphorylated AMPK alpha 1 and 2 (ab133448, rabbit monoclonal, used dilution: 1:1,000) were from ABCAM. Primary antibodies ( $($-actin, Myc-DDK) were visualized with ECL ${ }^{\mathrm{TM}}$ Plex goat anti-mouse IgG-Cy3 conjugate (Sigma C2181, used dilution: 1:2,500) using the Typhoon-Trio imaging system combined and Image Quant TL software 7.0 (GE Healthcare). Alternatively, they were visualized with peroxidase-coupled secondary antibodies (Sigma A8275, used dilution: 1:1,000), Super Signal West Pico (Pierce) (phosphorylated AMPK) or Super Signal West Femto (Pierce) (SLC35B1), and luminescence imaging using Fusion SL with the FUSION-CAP software 16.11 (PEQLAB). A home-made and affinity purified rabbit antipeptide antibody directed against the $\mathrm{COOH}$ terminal undecapeptide of human Sec62 protein (plus an aminoterminal cysteine) and Alexa Fluor594coupled secondary antibody from goat (Invitrogen A-11012, goat polyclonal, used dilution: 1:1,000) was used for fluorescence microscopy. The ApoSENSOR Bioluminescent Assay Kit was from BioVision and was used according to the manufacturer's instructions within an infinite M200 plate reader (TECAN). AICAR (5-aminoimidazole-4-carboxamide-1-ß-D-ribofuranoside), and emetine were from Sigma. We note that the full scans of blots and radioactive gels are shown in Supplementary Figs. 9-14. 


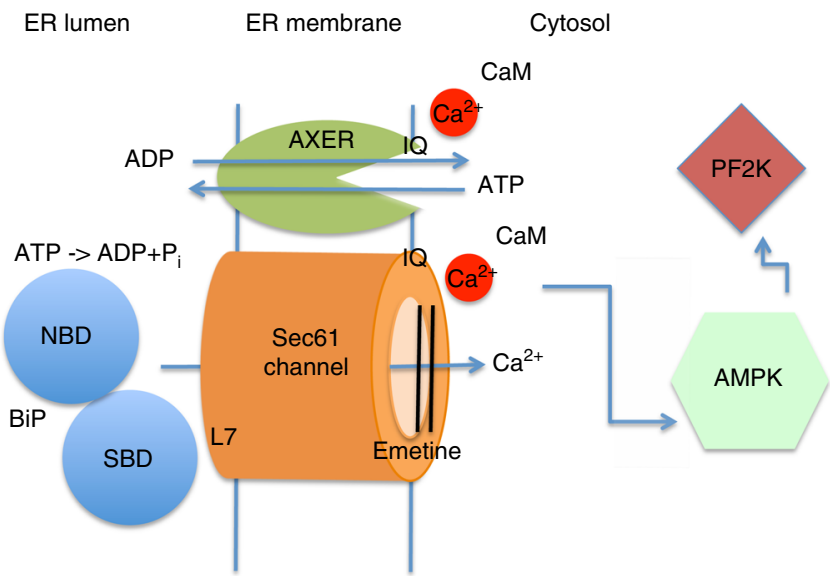

Fig. 9 ER low energy response (lowER) ensures a sufficient ATP supply to the human ER. AXER, ATP/ADP exchanger in the ER membrane = SLC35B1; AMPK AMP-activated protein kinase; CaM, Calmodulin; IQ, IQ motif $=\mathrm{Ca}^{2+}$-Calmodulin binding site ${ }^{27}$; emetine, inhibitor of polypeptide chain elongation at the ribosome; L7, ER lumenal loop 7 of Sec61 $\alpha=B$ BP binding site ${ }^{6}$; NBD, nucleotide binding domain of BiP; PF2K, 6-phosphofructo-2-kinase; $\mathrm{Pi}$, inorganic phosphate; $\mathrm{SBD}$, substrate binding domain of BiP. Notably, ATP production in HeLa cells relies mainly on glycolysis (in the cytosol) rather than on oxidative phosphorylation (in mitochondria) ${ }^{25}$. See text for details

Heterologous expression in E. coli and nucleotide import. The open reading frames for the respective carrier versions were Gateway cloned into the E. coli expression vector pET300 according to the manufacturer's instructions (Invitrogen). The corresponding primers are listed in Supplementary Table 3. The inserts were confirmed by sequencing. For heterologous expression, Rosetta 2 (DE3) pLysS cells (Novagen) were transformed with the expression vector constructs and grown at $37^{\circ} \mathrm{C}$ under aerobic conditions. At an optical density at $600 \mathrm{~nm}$ of 0.5 , heterologous protein synthesis was induced by the addition of $1 \mathrm{mM}$ isopropyl$\beta$-D-thiogalactopyranosid and the cells were harvested $1 \mathrm{~h}$ later by centrifugation (5 min, $5000 \times g, 15^{\circ} \mathrm{C}$ ). Non-induced cells served as the control. Recombinant protein synthesis and membrane insertion of the recombinant proteins were analyzed by Western blot. First, the pellet was resuspended in $1 \mathrm{mM}$ EDTA, 15\% glycerol, $10 \mathrm{mM}$ Tris- $\mathrm{HCl}, \mathrm{pH}$ 7.0, frozen in liquid nitrogen, and subsequently thawed at $37^{\circ} \mathrm{C}(5 \mathrm{~min})$. Autolysis was allowed at $37^{\circ} \mathrm{C}$ for $5 \mathrm{~min}$, and subsequent sonication on ice was used for cell disruption. Cell debris and protein aggregates were removed by centrifugation $\left(10,000 \times g, 15 \mathrm{~min}, 4^{\circ} \mathrm{C}\right)$. The membrane proteins in the supernatant were collected by ultracentrifugation $\left(100,000 \times g, 30 \mathrm{~min}, 4^{\circ} \mathrm{C}\right)$. Proteins were analyzed by SDS-PAGE, Western blotting, and immune detection with SLC35B1-specific antibodies. For analysis of the transport function of the recombinant proteins, the cell pellet was re-suspended in phosphate buffer $(50 \mathrm{mM}, \mathrm{pH} 7.0)$ directly after harvesting, and $100 \mu \mathrm{l}$ of the cells $\left(\mathrm{OD}_{600}\right.$ of 5.0$)$ were mixed with $100 \mu \mathrm{l}$ phosphate buffer supplemented with the indicated final concentrations of $\left[a^{32} \mathrm{P}\right]$-labeled ATP or $\mathrm{ADP}^{8}$. Transport was conducted at $30^{\circ} \mathrm{C}$ and terminated by removal of the external substrate via vacuum filtration and washing $(3 \times 4 \mathrm{ml}$ phosphate buffer washes). Radioactivity in the cell samples on the filters was quantified by scintillation counting (Tricarb 2500, CanberraPackard).

HeLa cell experiments. HeLa cells (DSM no. ACC 57) were obtained from the German Collection of Microorganisms and Cell Cultures, routinely tested for mycoplasma contamination by VenorGeM Mycoplasm Detection Kit (Biochrom AG, WVGM), and replaced every five years by a new batch. They were cultivated at $37^{\circ} \mathrm{C}$ in a humidified environment with $5 \% \mathrm{CO}_{2}$, in DMEM with $10 \%$ fetal bovine serum (FBS; Sigma) plus $1 \%$ penicillin and streptomycin. Cell growth was monitored using the Countess ${ }^{\circledast}$ Automated Cell Counter (Invitrogen) following the manufacturer's instructions. Alternatively, cell growth was monitored in real-time in an xCELLigence SP system (Roche Diagnostics) following the manufacturer's instruction $s^{37}$.

For gene silencing, $6.0 \times 10^{5} \mathrm{HeLa}$ cells were seeded per 6 -cm culture plate, followed by incubation in standard culture conditions. For SLC35B1 silencing, the cells were transfected with a final concentration of $20 \mathrm{nM}$ targeting siRNA (GGUACCCUGCCAUCAUCUAtt, GAGACUACCUCCACAUCAAtt (UTR)) (Qiagen) or with $20 \mathrm{nM}$ AllStars Negative Control siRNA (Qiagen) using HiPerFect Reagent (Qiagen) following the manufacturer's instructions. After $24 \mathrm{~h}$, the medium was changed and the cells were transfected a second time. Silencing efficiencies were evaluated by qRT-PCR (see below).
To rescue the phenotype after $S L C 35 B 1$ silencing with the corresponding human CDNA, cells were treated with SLC35B1-UTR siRNA as described above for $96 \mathrm{~h}$. Eight hours after the second transfection, the siRNA-treated cells were transfected with $4 \mu \mathrm{g}$ of the SLC35B1 expression plasmid using Fugene HD (Promega). Complementation was evaluated by qRT-PCR.

Super resolution microscopy. Cell-morphology and ER-morphology were analyzed by super-resolution fluorescence microscopy on an Elyra SIM (Carl Zeiss MicroImaging $)^{38}$. Cells were seeded on glass cover slips and treated as indicated. After $96 \mathrm{~h}$ the glass slides were removed and washed twice with cold PBS. Cells were fixed with $4 \%$ Paraformaldehyde for $20 \mathrm{~min}$ at $4{ }^{\circ} \mathrm{C}$. Fixed cells were permeabilized and blocked with PSS (PBS $+0.1 \%$ Saponin $+10 \%$ FCS) for $1 \mathrm{~h}$ at room temperature. To improve the antigen accessibility RNAse A (Roche) was added to a final concentration of $50 \mu \mathrm{g} / \mathrm{ml}$. After washing with PSS, indirect immunofluorescence staining with an affinity purified rabbit antipeptide antibody directed against Sec62 protein and Alexa Fluor594-coupled secondary antibody from goat was performed. Notably, the anti-Sec62 antibody is specific for Sec62 under denaturing, as well as native conditions (i.e., Western blot and fluorescence microscopy-signals were quenched after silencing of the SEC62 gene $)^{7,38}$. Cells were analyzed by microscopy on an Elyra SIM PS1 (Carl Zeiss-MicroImaging). The microscope was equipped with a Plan-Apochromat Oil DIC lense with $\times 63$ magnification and 1.4 numerical aperture (Carl Zeiss) and an $\mathrm{iXon}^{\mathrm{EM}}+885 \mathrm{EMCCD}$ camera (Andor Technology). Mounting medium was Roti ${ }^{\circledR}$-Mount FluorCare DAPI (Carl Roth), the oil was Immersol $518 \mathrm{~F}$ (Carl Zeiss).

Protein transport into semi-permeabilized cells. BiP activity was tested as ER protein import activity $26,38,39$. Precursor polypeptides were synthesized in reticulocyte lysate in the presence of $\left[{ }^{35} \mathrm{~S}\right]$ methionine for $16 \mathrm{~min}$ at $30^{\circ} \mathrm{C}$. After $5 \mathrm{~min}$ of incubation with puromycin (final concentration: $1 \mathrm{mM}$ ) at $30^{\circ} \mathrm{C}$, buffer or semipermeabilized cells, resulting in a final concentration of 12,800 cell equivalents/ $\mu \mathrm{l}$, were added and the incubation was continued for $30 \mathrm{~min}$. The cells had previously been treated with targeting or control siRNA for $96 \mathrm{~h}$. Semi-permeabilized-cells were prepared by digitonin treatment from identical cell numbers, adjusted according to $\mathrm{OD}_{280}$ in $2 \%$ SDS and, eventually, confirmed by SDS-PAGE and protein staining. All samples were analyzed by SDS-PAGE and phosphorimaging (Typhoon-Trio imaging system). Image Quant TL software 7.0 was used for quantifications.

Quantitative real-time PCR analysis. Total RNA was isolated from harvested cells using the RNA Blood Kit (Qiagen) following the manufacturer's instruc tions ${ }^{40}$. Reverse transcription was performed using the SuperScript VILO cDNA Synthesis Kit (Invitrogen, Thermo Fisher Scientific) and the cDNA was purified using the PCR Purification Kit (Qiagen). TaqMan ${ }^{\circledast}$ Gene Expression Assays (Applied Biosystems, Thermo Fisher Scientific) were used to perform quantitative real-time PCR of BIP (Hs99999174_m1), CHOP (Hs99999172_m1), and SLC35B1 (Hs00195184_m1) in a StepOne Plus 96-well system (Applied Biosystems). The $\triangle \mathrm{ct}$-values were calculated using $A C T B$ (Hs00357333_m1) as a standard, and the values were normalized based on control siRNA-treated cells.

Live-cell ATP imaging. Live-cell imaging of ATP in the cytosol and ER lumen was performed using the genetically encoded ATP sensors ATeam ${ }^{33,41}$ and ERAT4.0125, respectively. SLC35B1 silencing and expression plasmid based complementation in HeLa cells was performed for $96 \mathrm{~h}$ as described above. In addition, $24 \mathrm{~h}$ before measurement $(72 \mathrm{~h}$ after the first siRNA transfection), the cells were transfected with $1 \mu \mathrm{g}$ of the plasmids encoding the respective ATP sensor using Fugene HD. Then, $8 \mathrm{~h}$ after the last transfection, cells were seeded on $25 \mathrm{~mm}$ glass coverslips. Prior to the ATP measurements, the cells were incubated at room temperature for at least $2 \mathrm{~h}$ in loading buffer ${ }^{25}(135 \mathrm{mM} \mathrm{NaCl}, 5 \mathrm{mM} \mathrm{KCl}, 2 \mathrm{mM}$ $\mathrm{CaCl}_{2}, 1 \mathrm{mM} \mathrm{MgCl}$, $20 \mathrm{mM}$ HEPES, $2.6 \mathrm{mM} \mathrm{NaHCO}, 0.44 \mathrm{mM} \mathrm{KH}_{2} \mathrm{PO}_{4}, 0.34$ $\mathrm{mM} \mathrm{Na}_{2} \mathrm{HPO}_{4}, 10 \mathrm{mM}$ D-glucose). Coverslips were placed in a perfusion chamber, and imaging was performed at room temperature in Ringer's buffer $(145 \mathrm{mM}$ $\mathrm{NaCl}, 4 \mathrm{mM} \mathrm{KCl}, 2 \mathrm{mM} \mathrm{MgCl}, 2 \mathrm{mM} \mathrm{CaCl}$, $10 \mathrm{mM}$ Hepes, $10 \mathrm{mM}$ glucose, $\mathrm{pH}$ 7.4). Cells were imaged using a Cell Observer High Speed microscope (Zeiss) with a $\times 40$ oil Fluar objective (Zeiss) and an Evolve 512 EMCCD camera (Photometrics). The sensors were excited using $420 \mathrm{~nm}, 505 \mathrm{~nm}$, and white light LEDs (Colibri, Zeiss), while emission light was collected with CFP (Semrock HC) and YFP (Zeiss) single-band filters. Images of all channels were acquired every $3 \mathrm{~s}$, and FRET-ratios were calculated with AxioVision Software (Zeiss). Background fluorescence was subtracted, and the values were corrected for bleed-through and bleaching. Data were analyzed using Microsoft Excel 2013. $P$ values were determined using unpaired $t$-tests.

Live-cell $\mathbf{C a}^{2+}$ imaging. HeLa cells that were treated as described above were loaded with $4 \mu \mathrm{M}$ Fura-2 AM in DMEM and incubated for $30 \mathrm{~min}$ at room temperature ${ }^{6,27}$. The cells were washed twice and incubated at room temperature in $\mathrm{Ca}^{2+}$-free buffer $(140 \mathrm{mM} \mathrm{NaCl}, 5 \mathrm{mM} \mathrm{KCl}, 1 \mathrm{mM} \mathrm{MgCl}, 0.5 \mathrm{mM}$ EGTA, $10 \mathrm{mM}$ glucose in $10 \mathrm{mM}$ HEPES-KOH, pH 7.35). Ratiometric measurements were performed for 5 or 10 min using an iMIC microscope and the polychromator V (Till Photonics) with alternating excitation at $340 \mathrm{~nm}$ and $380 \mathrm{~nm}$ and measurement of 
the fluorescence emitted at $510 \mathrm{~nm}$. The microscope was equipped with a Fluar M27 lens with $\times 20$ magnification and 0.75 numerical aperture (Carl Zeiss) and an $\mathrm{iXon}^{\mathrm{EM}}+$ camera (Andor Technology). Images containing 50-55 cells/frame were sampled every $3 \mathrm{~s}$ using TILLvisION software (Till Photonics). Fura-2 signals were recorded as the F340/F380 ratio, where F340 and F380 correspond to the background-subtracted fluorescence intensities at $340 \mathrm{~nm}$ and $380 \mathrm{~nm}$, respectively. Data were analyzed using Microsoft Excel 2007. $P$ values were analyzed using unpaired $t$-tests.

Reconstitution of membrane proteins and ATP transport. Rough microsomes were isolated from canine or porcine pancreas and converted to ribosomedepleted microsomes (PKRM) by treatment with puromycin and high salt ${ }^{16}$ Alternatively, bacterial membranes from SLC35B1 expressing cells were isolated as described above. Proteoliposomes were prepared using $100 \mathrm{mg} / \mathrm{ml}$ phosphatidyl choline (type IV-S from soya bean; Sigma) and sonicated in $30 \mathrm{mM}$ potassium gluconate, $100 \mathrm{mM}$ Tricine- $\mathrm{NaOH}(\mathrm{pH} 7.5)^{42}$. Liposomes were subsequently preloaded with $10 \mathrm{mM}$ counter-exchange nucleotides (except when otherwise stated). PKRM and bacterial proteins were solubilized with $1 \%$ Triton X-100 and $1 \%$ dodecylmatoside, resepectively, for $1 \mathrm{~min}$ on ice. The samples were centrifuged $\left(1 \mathrm{~min}, 15,800 \times g\right.$ at $\left.4{ }^{\circ} \mathrm{C}\right)$ and the supernatants were rapidly combined with the liposomes and mixed vigorously (resulting detergent concentration $0.1 \%$ ). The vigorously mixed suspension was subsequently transferred to liquid nitrogen to allow incorporation of the proteins into the vesicles after thawing the sample at $4{ }^{\circ} \mathrm{C}$ (freeze-thaw method). The thawed proteoliposomes were sonicated for $30 \mathrm{~s}$ ( $20 \%$ line voltage, $20 \%$ duty cycle) on ice. Unincorporated solution was removed by passing the proteoliposomes over NAP-5 gel filtration columns pre-equilibrated with $150 \mathrm{mM}$ potassium gluconate, 10 $\mathrm{mM}$ Tricine- $\mathrm{NaOH}, \mathrm{pH} 7.5$ at $4{ }^{\circ} \mathrm{C}$. The eluted proteoliposomes $(1 \mathrm{ml})$ were immediately used for transport measurements. Transport at $30^{\circ} \mathrm{C}$ was started by adding $100 \mu \mathrm{l}$ proteoliposomes to $100 \mu \mathrm{l}$ transport medium $(150 \mathrm{mM}$ potassium gluconate, $10 \mathrm{mM}$ Tricine- $\mathrm{NaOH}, \mathrm{pH} 7.5)$ containing the indicated [ $\left.{ }^{32} \mathrm{P}\right]$-labeled substrates. Uptake occurred at the indicated time spans and was stopped by transferring the liposomes to a $1 \mathrm{ml}$ Dowex AG-1 ( $\mathrm{Cl}^{-}$form; $\left.100-200 \mathrm{mesh}\right)$ column pre-equilibrated with $200 \mathrm{mM}$ Tricine- $\mathrm{NaOH} \mathrm{pH} 7.5$. By washing the columns with $3 \times 500 \mu \mathrm{l}$ ice cold equilibration medium the proteoliposomes were eluted and external radioactivity could be removed. Imported radioactivity was quantified in a scintillation counter.

AXER modeling and ATP docking. The comparative atomic model of SLC35B was modeled using two different computational approaches: Phyre $2^{34}$ and Raptor $\mathrm{X}^{43}$. The structure predicted by Phyre2 is very similar to that of the DMT superfamily transporter YddG (PDB ID: 5I20) with an RMSD of $0.4 \AA$. On the other hand, the structure of SLC35B1 predicted by RaptorX was based on that of the triose-phosphate/phosphate translocator (PDB ID: 5 Y78) ${ }^{44}$. It has a RMSD of $4.8 \AA$ of RMSD compared to the template. The two models show a similar topological arrangement of the transmembrane helices. The RMSD difference between the two models is $6.97 \AA$. A residue contact map was derived by the RaptorX Contact Prediction method ${ }^{45}$ to validate the two models obtained in the previous step. The validation showed that $59 \%$ of the predicted contact pairs are fulfilled by the Phyre 2 model (only considering contacts with more than $90 \%$ probability in the contact map). For comparison, $81 \%$ of the predicted contact pairs are fulfilled by the RaptorX model. However, as the RaptorX model adopts a closed structure, we considered the open Phyre 2 to be more promising for a ligand docking study.

Hence, the model obtained by Phyre2 was subjected to energy minimization by the NAMD package to relax the side-chain atoms. Subsequently, the minimized structure was subjected to the ProSa-web server for structure validation. The ProSa $Z$-score of -3.03 is in the typical range or scores found for experimentally determined structures of protein chains. The ATP molecular structure was retrieved from the PubChem database. Protonation states at neutral $\mathrm{pH}$ were assigned with the Babel software. The resulting structure of ATP had the expected total charge of $-4 \mathrm{e}$. After setting up protein and ligand, docking runs were carried out using the AutoDock4 program $\left(2.5 \times 10^{6}\right.$ energy evaluations and $27 \times 10^{3}$ generations) to scan for energetically favorable conformations of the ligand inside the protein pocket.

Data availability. Source files for Western blots were deposited at Mendeley (https://doi.org/10.17632/b49tkfjcm5.1). In addtion, all data are available from the authors.

Received: 14 December 2017 Accepted: 6 August 2018

Published online: 28 August 2018

\section{References}

1. Haas, I. \& Wabl, M. Immunoglobulin heavy chain binding protein. Nature 306, 387-389 (1983).
2. Hendershot, L. M., Ting, J. \& Lee, A. S. Identity of the immunoglobulin heavy chain binding protein with the 78,000-dalton glucose-regulated protein and the role of posttranslational modifications in its binding function. Mol. Cell. Biol. 8, 4250-4256 (1988).

3. Lievremont, J. P., Rizzuto, R., Hendershot, L. \& Meldolesi, J. BiP, a major chaperone protein of the endoplasmic reticulum lumen, plays a direct and important role in the storage of the rapidly exchanging pool of $\mathrm{Ca}^{2+}$. J. Biol. Chem. 272, 30873-30879 (1997).

4. Hamman, B. D., Hendershot, L. M. \& Johnson, A. E. BiP maintains the permeability barrier of the ER membrane by sealing the luminal end of the translocon pore before and early in translocation. Cell 92, 747-758 (1998).

5. Bertolotti, A., Zhang, Y., Hendershot, L. M., Harding, H. P. \& Ron, D. Dynamic interaction of BiP and ER stress transducers in the unfolded-protein response. Nat. Cell Biol. 2, 326-332 (2000).

6. Schäuble, N. et al. BiP-mediated closing of the Sec61 channel limits $\mathrm{Ca}^{2+}$ leakage from the ER. EMBO J. 31, 3282-3296 (2012).

7. Tyedmers, J., Lerner, M., Wiedmann, M., Volkmer, J. \& Zimmermann, R. Polypeptide chain binding proteins mediate completion of cotranslational protein translocation into the mammalian endoplasmic reticulum. EMBO Rep. 4, 505-510 (2003).

8. Leroch, M. et al. Identification of a novel adenine nucleotide transporter in the endoplasmic reticulum of Arabidopsis. Plant Cell 20, 438-451 (2008).

9. Chu, L. et al. Shuttling of (deoxy-) nucleotides between compartments of the diatom Phaeodactylum tricornutum. New Phytol. 213, 193-205 (2017).

10. Jack, D. L., Yang, N. M. \& Saier, M. H. Jr The drug/metabolite transporter superfamily. Eur. J. Biochem. 268, 3620-3639 (2001).

11. Schlessinger, A., Yee, S. W., Sali, A. \& Giacomoni, K. M. SLC classification: an update. Clin. Pharma. Ther. 94, 19-22 (2013).

12. Nakanishi, H. et al. Hut I proteins indentified in Saccharomyces cerevisiae and Schizosaccharomyces pombe are functional homologues involved in the proteinfolding process at the endoplasmic reticulum. Yeast 18, 543-554 (2001).

13. Dejima, K. et al. The ortholog of human solute carrier family 35 member B1 (UDP-galactose transporter-related protein 1) is involved in maintenance of ER homeostasis and essential for larval development in Caenorhabditis elegans. FASEB J. 23, 2215-2225 (2009).

14. Reyes, F. et al. AtUTr1, a UDP-glucose/UDP-galactose transporter from Arabidopsis thaliana, is located in the endoplasmic reticulum and upregulated by the unfolded protein response. J. Biol. Chem. 281, 9145-9151 (2006).

15. Tsuchiya, H. et al. Structural basis for amino acid export by DMT superfamily transporter YddG. Nature 534, 417-420 (2016).

16. Wirth, A. et al. The Sec61p complex is a dynamic precursor activated channel. Mol. Cell 12, 261-268 (2003).

17. Hein, M. Y. et al. A human interactome in three quantitative dimensions organized by stoichiometrics and abundances. Cell 163, 712-723 (2015).

18. Haßdenteufel, $\mathrm{S}$. et al. hSND2 protein represents an alternative targeting factor to the endoplasmic reticulum in human cells. FEBS Lett. 591, 3211-3224 (2017)

19. Haferkamp, I. et al. A candidate $\mathrm{NAD}^{+}$transporter in an intracellular bacterial symbiont related to Chlamydiae. Nature 432, 622-625 (2004).

20. Mayinger, P., Bankaitis, V. A. \& Meyer, D. I. Sac1p mediates the adenosine triphosphate transport into yeast endoplasmic reticulum that is required for protein translocation. J. Cell. Biol. 131, 1377-1386 (1995).

21. Kochendörfer, K. U., Then, A. R., Kearns, B. G., Bankaitis, V. A. \& Mayinger, P. Saclp plays a crucial role in microsomal ATP transport, which is distinct from its function in Golgi phospholipid metabolism. EMBO J. 18, 1506-1515 (1999).

22. Guillén, E. \& Hirschberg, C. B. Transport of adenosine triphosphate into endoplasmic reticulum proteoliposomes. Biochemistry 34, 5472-5476 (1995).

23. Shin, S. J., Lee, W. K., Lim, H. W. \& Park, J.-S. Characterization of the ATP transporter in the reconstituted rough endoplasmic reticulum proteoliposomes. Biochem. Biophys. Acta 1468, 55-62 (2000).

24. Nishito, H. CHOP is a multifunctional transcription factor in the ER stress response. J. Biochem. 151, 217-219 (2012).

25. Vishnu, N. et al. ATP increases within the lumen of the endoplasmic reticulum upon intracellular $\mathrm{Ca}^{2+}$ release. Mol. Biol. Cell. 25, 368-379 (2014).

26. Haßdenteufel, S. et al. Chaperone-mediated Sec61 channel gating during ER import of small presecretory proteins overcomes Sec61 inhibitor-reinforced energy barrier. Cell Rep. 23, 1373-1386 (2018).

27. Erdmann, F. et al. Interaction of calmodulin with Sec61a limits $\mathrm{Ca}^{2+}$ leakage from the endoplasmic reticulum. EMBO J. 30, 17-31 (2011).

28. Flourakis, M., Van Coppenolle, F., Lehen'kyi, V., Beck, B. \& Skryma, R. Passive calcium leak via translocon is a first step for iPLA2-pathway regulated store operated channels activation. FASEB J. 20, 1215-1217 (2006). 
29. Alder, N. N., Shen, Y., Brodsky, J. L., Hendershot, L. M. \& Johnson, A. E. The molecular mechanisms underlying BiP-mediated gating of the Sec61 translocon of the endoplasmic reticulum. J. Cell. Biol. 168, 389-399 (2005).

30. Preston, A. M. \& Hendershot, L. M. Examination of a second node of translational control in the unfolded protein response. J. Cell. Sci. 126, 4253-4261 (2013).

31. Long, Y. C. \& Zierath, J. R. AMP-activated protein kinase signaling in metabolic regulation. J. Clin. Invest. 116, 1776-1783 (2006).

32. Jeon, S.-M. Regulation and function of AMPK in physiology and diseases. Exp. Mol. Med. 48, e245 (2016).

33. Imamura, H. et al. Visualization of ATP levels inside living cells with fluorescence resonance energy transfer-based genetically encoded indicators. Proc. Natl Acad. Sci. USA 106, 15651-15656 (2009).

34. Kelley, L. A., Mezulis, S., Yates, C. M., Wass, M. N. \& Sternberg, M. The Phyre2 web portal for protein modeling, prediction and analysis. Nat. Prot. 10, 845-858 (2015).

35. Klingenberg, M. The ADP and ATP transport in mitochondria and its carrier. Biochem. Biophys. Acta 1778, 1978-2021 (2008).

36. Ponsero, A. J. et al. Endoplasmic reticulum transport of glutathione by Sec61 is regulated by Ero1 and BiP. Mol. Cell 67, 962-973 (2017).

37. Linxweiler, $M$. et al. Targeting cell migration and the endoplasmic reticulum stress response with calmodulin antagonists: a clinically tested small molecule phenocopy of SEC62 gene silencing in human tumor cells. BMC Cancer 13, 574 (2013).

38. Lang, S. et al. Different effects of Sec61a, Sec62 and Sec63 depletion on transport of polypeptides into the endoplasmic reticulum of mammalian cells. J. Cell Sci. 125, 1958-1969 (2012).

39. Johnson, N. et al. TRC40 can deliver short secretory proteins to the Sec61 translocon. J. Cell Sci. 125, 3612-3620 (2012).

40. Schorr, S. et al. Co-chaperone specificity in gating of the polypeptide conducting channel in the membrane of the human endoplasmic reticulum. J. Biol. Chem. 290, 18621-18635 (2015).

41. Roesch, A. et al. Overcoming intrinsic multidrug resistance in melanoma by blocking the mitochondrial respiratory chain of slow-cycling JARID1B high cells. Cancer Cell. 23, 811-825 (2013).

42. Möhlmann, T. et al. ADP-glucose drives starch synthesis in isolated maize endosperm amyloplasts: characterization of starch synthesis and transport properties across the amyloplast envelope. Biochem. J. 324, 503-509 (1997).

43. Källberg, M. et al. Template-based protein structure modeling using the RaptorX web server. Nat. Protoc. 7, 1511-1522 (2012).

44. Lee, Y. et al. Structure of the triose-phosphate/phosphate translocator reveals the basis of substrate specificity. Nat. Plants 3, 825-832 (2017).

45. Wang, S., Sun, S., Li, Z., Zhang, R. \& Xu, J. Accurate de novo prediction of protein contact map by ultra-deep learning model. PLOS Comp. Biol. https:// doi.org/10.1371/journal.pcbi.1005324 (2017).

46. Boß, M. et al. AMPK-independent inhibition of human macrophage ER stress response by AICAR. Sci. Rep. 6, 32111 (2016).

\section{Acknowledgements}

The authors are grateful to Dr. Joe Casey (MPDRG, University of Alberta, Edmonton, Canada) for stimulating discussions. I.B. was supported by the DFG (SFB 1027, SFB 1190 , and BO3643/3-2), A.C., J.R., and M.H. by the DFG (SFB 894), R.Z. by the DFG (SFB 894 and IRTG1830), and H.E.N. by the DFG (IRTG 1830).

\section{Author contributions}

M.-C.K. performed all SLC35B1 knockdowns in HeLa cells, and most subsequent analyses including the $\mathrm{Ca}^{2+}$ imaging experiments under the supervision of S.L. and A.C., and the SIM microscopy under the supervision of E.K. and J.R. K.Z. performed the ATP imaging experiments under the supervision of I.B. and M.H. S.S. performed the qPCR experiments I.H. and M.L. performed all ATP transport experiments in E. coli and proteoliposomes with the help of P.K. under the supervision of H.E.N. J.A. performed the curve fittings with I.H. C.F.-T. carried out MS. M.J. contributed ER membranes, structural predictions, and peptide array binding data. D.N. performed modeling and docking analyses under supervision by V. H. R.Z. designed the study and wrote the manuscript with I.B., M.H., H.E.N., S.L., and I.H All authors discussed the results and approved the final manuscript.

\section{Additional information}

Supplementary Information accompanies this paper at https://doi.org/10.1038/s41467018-06003-9.

Competing interests: The authors declare no competing interests.

Reprints and permission information is available online at http://npg.nature.com/ reprintsandpermissions/

Publisher's note: Springer Nature remains neutral with regard to jurisdictional claims in published maps and institutional affiliations.

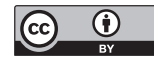

Open Access This article is licensed under a Creative Commons Attribution 4.0 International License, which permits use, sharing, adaptation, distribution and reproduction in any medium or format, as long as you give appropriate credit to the original author(s) and the source, provide a link to the Creative Commons license, and indicate if changes were made. The images or other third party material in this article are included in the article's Creative Commons license, unless indicated otherwise in a credit line to the material. If material is not included in the article's Creative Commons license and your intended use is not permitted by statutory regulation or exceeds the permitted use, you will need to obtain permission directly from the copyright holder. To view a copy of this license, visit http://creativecommons.org/ licenses/by/4.0/.

(C) The Author(s) 2018 